\title{
AN ASYMPTOTIC ESTIMATE FOR HEIGHTS OF ALGEBRAIC SUBSPACES
}

\author{
JEFFREY LIN THUNDER
}

\begin{abstract}
We count the number of subspaces of affine space with a given dimension defined over an algebraic number field with height less than or equal to $B$. We give an explicit asymptotic estimate for the number of such subspaces as $B$ goes to infinity, where the constants involved depend on the classical invariants of the number field (degree, discriminant, class number, etc.). The problem is reformulated as an estimate for the number of lattice points in a certain bounded domain.
\end{abstract}

\section{INTRODUCTION}

The purpose of a height is to give an explicit quantification for how "complicated" an object is. If $S$ is a one-dimensional subspace of $\mathbb{Q}^{n}$, the distance between integral points in $S$ is one way to measure how "complicated" $S$ is. For such a subspace, the integral points are of the form $\mathbb{Z} \mathbf{p}$, where $\mathbf{p}$ is a primitive lattice point, i.e., a point in $\mathbb{Z}^{n}$ with coprime coorcinates. We define the height of $S, H(S)$, to be the Euclidean norm of the point p. Now two primitive lattice points, $\mathbf{p}_{1}$ and $\mathbf{p}_{2}$, will be integral points in the same subspace if and only if $\mathbf{p}_{1}= \pm \mathbf{p}_{2}$. Thus, the number of one-dimensional subspaces of $\mathbb{Q}^{n}$ with height $\leq B$ is one-half the number of primitive lattice points in the ball of radius $B$. By [5, Theorem 459], this number is asymptotically

$$
\frac{V(n)}{2 \zeta(n)} B^{n}
$$

as $B \rightarrow \infty$, where $\zeta$ is the Riemann zeta function and $V(n)$ is the volume of the unit ball in $\mathbb{R}^{n}$.

The present paper is concerned with a generalization of this asymptotic formula, where the one-dimensional subspaces of $\mathbb{Q}^{n}$ are replaced by $d$-dimensional subspaces of $K^{n}$, where $0<d<n$ and $K$ is an algebraic number field. It turns out that there is a natural way to define the height of a $d$ dimensional subspace of $K^{n}$ (see the end of this introduction). Fix the field $K$ and let $M(n, d, B)$ be the number of $d$-dimensional subspaces $S \subset K^{n}$ with $H(S) \leq B$.

Theorem 1. As $B \rightarrow \infty$, we have

$$
M(n, d, B)=a(n, d) B^{n}+O\left(B^{n-b(n, d)}\right),
$$

Received by the editors June 1, 1990.

1980 Mathematics Subject Classification (1985 Revision). Primary 11G99; Secondary 11H16. 
where $a(n, d)$ and $b(n, d)$ are explicit positive constants and the constant implicit in the $O$ notation depends only on $K$ and $n$.

The values of the constants above are as follows:

$$
b(n, d)=\max \left\{\frac{1}{\kappa d}, \frac{1}{\kappa(n-d)}\right\},
$$

where $\kappa$ is the degree of $K$ over $\mathbb{Q}$. Write $\kappa=r_{1}+2 r_{2}$, where $r_{1}$ is the number of real embeddings of $K$ into the complex numbers $\mathbb{C}$, and $r_{2}$ is the number of pairs of complex conjugate embeddings. Let $\delta$ be the discriminant, $R$ the regulator, $h$ the class number, and $w$ the number of roots of unity of $K$. Further, let $\zeta_{K}$ be the Dedekind zeta function of $K$, and introduce the function $V_{2}(n)=V(2 n)$. Given a function $f$ defined for $n=2,3, \ldots$ and having nonzero values, introduce the generalized binomial symbol

$$
\left(\left.f\right|_{d} ^{n}\right)=\frac{f(n) f(n-1) \cdots f(n-d+1)}{f(2) f(3) \cdots f(d)}
$$

defined for $0<d<n$, with $f(2) f(3) \cdots f(d)$ to be interpreted as 1 when $d=1$. Note that $\left(\left.f\right|_{d} ^{n}\right)=\left(\left.f\right|_{n-d} ^{n}\right)$. With this notation,

$$
a(n, d)=\frac{h R}{w n}\left(\frac{2^{r_{2}}}{\sqrt{|\delta|}}\right)^{(n-d) d+1}\left(\begin{array}{l}
n \\
d
\end{array}\right)^{r_{1}+r_{2}}\left(\left.V\right|_{d} ^{n}\right)^{r_{1}}\left(\left.V_{2}\right|_{d} ^{n}\right)^{r_{2}}\left(\left.\zeta_{K}\right|_{d} ^{n}\right)^{-1} .
$$

Note the symmetry of the result: $M(n, d, B)=M(n, n-d, B)$. It is known (see $[11$, p. 433]) that

$$
H(S)=H\left(S^{\perp}\right),
$$

where $S^{\perp}$ is the "orthogonal complement" of the space $S$ in $K^{n} ; S^{\perp}$ consists of all vectors $\alpha=\left(\alpha_{1}, \alpha_{2}, \ldots, \alpha_{n}\right) \in K^{n}$ such that

$$
\boldsymbol{\alpha} \cdot \boldsymbol{\beta}=\alpha_{1} \beta_{1}+\alpha_{2} \beta_{2}+\cdots+\alpha_{n} \beta_{n}=0
$$

for all $\beta \in S$. Since $\operatorname{dim}_{K} S^{\perp}=n-\operatorname{dim}_{K} S$, this explains the symmetry.

Theorem 1 generalizes two previous results. W. Schmidt [12] proved Theorem 1 in the special case $K=\mathbb{Q}$. Also, S. Schanuel [10] has done the case $d=1$ with a general number field, although with a slightly different definition of height. When $K=\mathbb{Q}$ and $d=1$, we have

$$
a(n, 1)=\frac{V(n)}{2 \zeta(n)},
$$

so that our theorem implies the asymptotic formula (1).

Theorem 1 has an interpretation in the context of algebraic geometry. As is well known, subspaces of given dimension $d$ in $K^{n}$ correspond to points on a Grassmann variety (see below). Our result may thus be interpreted as counting the number of points of height $\leq B$ on this (projective) variety. Recently, Franke, Manin, and Tschinkel [4] have studied the asymptotic behavior of the number of points with height $\leq B$ on Fano varieties (i.e., those varieties for which the anticanonical bundle $\omega^{-1}$ is ample) which includes Grassmann varieties as a specific example. However, they provide no formula for the constants in the asymptotic relations. Also, they use the Arakelov height, so the exponent on $B$ is different. There is no method for counting integral points of height 
$\leq B$ on a general variety. In our case we succeed because of the definition of the Grassmann variety in terms of subspaces.

We end this introduction with the definition of the height of subspaces. Such a definition had first been given in [11]. Let $M(K)$ be the set of places of $K$, and let $\alpha \mapsto \alpha^{(i)} \quad(1 \leq i \leq \kappa)$ denote the embeddings of $K$ into the complex numbers, ordered so that the first $r_{1}$ are real and $\alpha^{\left(i+r_{2}\right)}=\overline{\alpha^{(i)}}$ for $r_{1}+1 \leq i \leq r_{1}+r_{2}$, where $\bar{\alpha}$ denotes the complex conjugate of the number $\alpha$.

To each nonarchimedean place $v \in M(K)$, let $|\cdot|_{v}$ be the corresponding absolute value on $K$, normalized to extend the $p$-adic absolute value on $\mathbb{Q}$, where $v$ lies above the rational prime $p$. We also have the absolute value, $|\cdot|_{v}$, for each archimedean place $v \in M(K)$, defined by

$$
|\alpha|_{v}= \begin{cases}\left|\alpha^{(i)}\right| & \text { for } 1 \leq i \leq r_{1}, \\ \left|\alpha^{(i)} \alpha^{\left(i+r_{2}\right)}\right|^{1 / 2} & \text { for } r_{1}<i \leq r_{1}+r_{2},\end{cases}
$$

where $v$ corresponds to the embedding $\alpha \mapsto \alpha^{(i)}$ and $|\cdot|$ denotes the usual absolute value on $\mathbb{R}$.

For each place $v \in M(K)$, let $n_{v}$ be the local degree. We have the product formula (see [8, Chapter 5]):

$$
\prod_{v \in M(K)}|a|_{v}^{n_{v}}=1
$$

for all $a \in K^{*}=K \backslash\{0\}$.

Given a vector $\boldsymbol{\alpha}=\left(\alpha_{1}, \alpha_{2}, \ldots, \alpha_{n}\right) \in K^{n}$ and a $v \in M(K)$, put

$$
\|\boldsymbol{\alpha}\|_{v}= \begin{cases}\left(\sum_{i=1}^{n}\left|\alpha_{i}\right|_{v}^{2}\right)_{v}^{n_{v} / 2} & \text { if } v \text { is archimedean } \\ \max _{1 \leq i \leq n}\left|\alpha_{i}\right|_{v}^{n_{v}} & \text { otherwise. }\end{cases}
$$

Note that, by the product formula and the definitions,

$$
\prod_{v \in M(K)}\|a \boldsymbol{\alpha}\|_{v}=\prod_{v \in M(K)}|a|_{v}^{n_{v}} \cdot \prod_{v \in M(K)}\|\boldsymbol{\alpha}\|_{v}=\prod_{v \in M(K)}\|\boldsymbol{\alpha}\|_{v}
$$

for all $a \in K^{*}$. Thus, we may define the height of a one-dimensional subspace, $K \boldsymbol{\alpha} \subset K^{n}, \boldsymbol{\alpha} \neq \mathbf{0}$, by

$$
H(K \boldsymbol{\alpha})=H(\boldsymbol{\alpha})=\prod_{v \in M(K)}\|\boldsymbol{\alpha}\|_{v} .
$$

There are other definitions of height. We use this one here in order to simplify our argument (in particular, the statement of Theorem 2 below).

Let $\alpha \in K^{n}$ be as above and denote by [ $\alpha$ ] the fractional ideal generated by the components, $\alpha_{1}, \alpha_{2}, \ldots, \alpha_{n}$ of $\alpha$. It is well known (see, for example, $[8$, Chapter 5]) that

$$
\prod_{v \in M_{0}(K)}\|\boldsymbol{\alpha}\|_{v}=N([\boldsymbol{\alpha}])^{-1},
$$

where $M_{0}(K)$ denotes the nonarchimedean places of $K$ and $N$ is the norm of the ideal.

We thus have

$$
H(\boldsymbol{\alpha})=N([\boldsymbol{\alpha}])^{-1} \cdot \prod_{i=1}^{\kappa}\left\|\boldsymbol{\alpha}^{(i)}\right\|=N([\boldsymbol{\alpha}])^{-1} \cdot \prod_{i=1}^{r_{1}+r_{2}}\left\|\boldsymbol{\alpha}^{(i)}\right\|^{e_{i}},
$$


where $\|\cdot\|$ denotes the usual norm on $\mathbb{C}^{n}$ :

$$
\|\boldsymbol{\alpha}\|=\left(\sum_{i=1}^{n} \alpha_{i} \overline{\alpha_{i}}\right)^{1 / 2},
$$

and

$$
e_{i}= \begin{cases}1 & \text { if } i \leq r_{1}, \\ 2 & \text { if } i>r_{1}\end{cases}
$$

It will be convenient to write

$$
H_{\infty}(\boldsymbol{\alpha})=\prod_{i=1}^{r_{1}+r_{2}}\left\|\boldsymbol{\alpha}^{(i)}\right\|^{e_{i}},
$$

so that $H(\boldsymbol{\alpha})=N([\boldsymbol{\alpha}])^{-1} H_{\infty}(\boldsymbol{\alpha})$.

Now suppose $S$ is a $d$-dimensional subspace of $K^{n}$, where $1 \leq d<n$. If $\boldsymbol{\alpha}_{1}, \boldsymbol{\alpha}_{2}, \ldots, \boldsymbol{\alpha}_{d}$ are a basis for $S$ over $K$, we can form the wedge product (see [7, Chapter 7]):

$$
\boldsymbol{\alpha}_{1} \wedge \boldsymbol{\alpha}_{2} \wedge \cdots \wedge \boldsymbol{\alpha}_{d} \in K^{\left(\begin{array}{c}
n \\
d
\end{array}\right),}
$$

where $\left(\begin{array}{l}n \\ d\end{array}\right)$ is the binomial coefficient, $\frac{n !}{d !(n-d) !}$. Also, we have

$$
\boldsymbol{\alpha}_{1} \wedge \boldsymbol{\alpha}_{2} \wedge \cdots \wedge \boldsymbol{\alpha}_{d} \in K^{*}\left(\boldsymbol{\beta}_{1} \wedge \boldsymbol{\beta}_{2} \wedge \cdots \wedge \boldsymbol{\beta}_{d}\right)
$$

if and only if the $\alpha$ 's and the $\beta$ 's span the same subspace. (For proofs, see [7].) Thus, the wedge product will give a one-to-one (though not generally onto) mapping of $d$-dimensional subspaces of $K^{n}$ to one-dimensional subpaces of

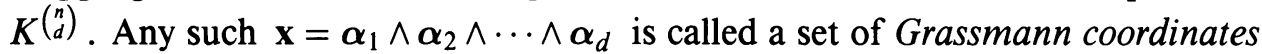
of $S$. Such an $\mathbf{x}$ is determined up to a scalar multiple. We define the height of the subspace $S$ as $H(S)=H(\mathbf{x})$. Finally, define

$$
H(\{\mathbf{0}\})=H\left(K^{n}\right)=1 .
$$

A vector in $K^{\left(\begin{array}{l}n \\ d\end{array}\right)}$ is called decomposable if it is of the form $\boldsymbol{\alpha}_{1} \wedge \boldsymbol{\alpha}_{2} \wedge \cdots \wedge \boldsymbol{\alpha}_{d}$ for vectors $\boldsymbol{\alpha}_{1}, \boldsymbol{\alpha}_{2}, \ldots, \boldsymbol{\alpha}_{d}$ in $K^{n}$. Such vectors, for a fixed $d$, form a variety, called the (affine) Grassmannian or Grassmann variety. Given a subspace $S$, we may choose [ $\mathbf{x}]$, where $\mathbf{x}$ is a set of Grassmann coordinates of $S$, up to multiplication by a principal ideal. One may fix an ideal $\mathfrak{A}$ in each ideal class and count the number of points $\mathbf{x}$ on the Grassmann variety with height $\leq B$ and with $[\mathbf{x}]=\mathfrak{A}$. After dividing out by the action of the units, this translates loosely into counting "lattice points" on the variety. This is how S. Schanuel proceeds in [10], when the Grassmann variety is just $K^{n}$.

Last, but not least, the author would like to take this opportunity to thank his thesis advisor, W. Schmidt, for the help and encouragment he has given.

\section{A SKEWED HEIGHT AND A REDUCTION}

The definition of height for a subspace of dimension greater than one given in $\S 1$ seems somehow removed from the idea that the height should measure how "complicated" it is. Recall that we started with a one-dimensional subspace of $\mathbb{Q}^{n}$ and used the distance between integral points of the subspace as the height. In this case, the integral points are of the form $\mathbb{Z p}$, where $\mathbf{p}$ is a primitive lattice point. In other words, the integral points of a one-dimensional 
subspace of $\mathbb{Q}^{n}$ form a sublattice of dimension 1 of $\mathbb{Z}^{n}$ (see below). Similarly, one sees that the integral points of a $d$-dimensional subspace form a sublattice of dimension $d$. Thus, the determinant of the lattice of integral points of the subspace gives a measure of how "complicated" the subspace is. In fact, this is what our definition of height turns out to be in the special case $K=\mathbb{Q}$.

Now for a general number field, the integral points of a $d$-dimensional subspace will form an $\mathfrak{O}_{K}$-module, where $\mathfrak{O}_{K}$ is the ring of integers in $K$. But this module will not in general be free, so one cannot apply the idea of a determinant without more work. Our first task is to get an alternative definition of height which generalizes the ideas above.

For $\mathbf{X} \in \mathbb{R}^{n r_{1}} \oplus \mathbb{C}^{2 n r_{2}}$ we write

$$
\mathbf{X}=\left(\mathbf{x}_{1}, \mathbf{x}_{2}, \ldots, \mathbf{x}_{\kappa}\right),
$$

where

$$
\mathbf{x}_{i} \in \begin{cases}\mathbb{R}^{n} & \text { for } 1 \leq i \leq r_{1}, \\ \mathbb{C}^{n} & \text { for } r_{1}<i \leq \kappa .\end{cases}
$$

Let $\mathbb{E}^{n \kappa} \subset \mathbb{R}^{n r_{1}} \oplus \mathbb{C}^{2 n r_{2}}$ be given by the set of points satisfying

$$
\mathbf{x}_{i+r_{2}}=\overline{\mathbf{x}}_{i} \quad \text { for } r_{1}<i \leq r_{1}+r_{2} .
$$

For $\mathbf{X}$ and $\mathbf{Y}$ in $\mathbb{E}^{n \kappa}$, we define the inner product of $\mathbf{X}$ and $\mathbf{Y}$ to be $\mathbf{X} \cdot \overline{\mathbf{Y}}$, the usual inner product in $\mathbb{C}^{n \kappa}$. Thus, for $\mathbf{X}=\left(\mathbf{x}_{1}, \mathbf{x}_{2}, \ldots, \mathbf{x}_{\kappa}\right)$ and $\mathbf{Y}=$ $\left(\mathbf{y}_{1}, \mathbf{y}_{2}, \ldots, \mathbf{y}_{\kappa}\right)$ in $\mathbb{E}^{n \kappa}$,

$$
\mathbf{X} \cdot \overline{\mathbf{Y}}=\sum_{i=1}^{r_{1}} \mathbf{x}_{i} \cdot \mathbf{y}_{i}+\sum_{i=r_{1}+1}^{r_{1}+2 r_{2}} \mathbf{x}_{i} \cdot \overline{\mathbf{y}_{i}} .
$$

One easily verifies that, under this inner product, $\mathbb{E}^{n \kappa}$ is a Euclidean vector space of dimension $n \kappa$. In particular, $\mathbf{X} \cdot \overline{\mathbf{Y}}$ is real and $\mathbf{X} \cdot \overline{\mathbf{Y}}=\mathbf{Y} \cdot \overline{\mathbf{X}}$.

We embed $K^{n}$ into $\mathbb{E}^{n \kappa}$ as follows: let $\rho: K^{n} \rightarrow \mathbb{E}^{n \kappa}$ be defined by

$$
\rho(\boldsymbol{\alpha})=\left(\boldsymbol{\alpha}^{(1)}, \boldsymbol{\alpha}^{(2)}, \ldots, \boldsymbol{\alpha}^{(\kappa)}\right),
$$

where the maps $\alpha \mapsto \alpha^{(i)}$ denote the embeddings of $K$ into $\mathbb{C}$, ordered as above.

By a lattice $\Lambda$ in a Euclidean space $\mathbb{E}$, we will mean a discrete subgroup of the additive group $\mathbb{E}$. The dimension of the lattice $\Lambda$ is the dimension of the subspace spanned by $\Lambda$. Suppose $\mathbf{X}_{1}, \mathbf{X}_{2}, \ldots, \mathbf{X}_{m}$ are a basis for $\Lambda$, so that

$$
\Lambda=\bigoplus_{i=1}^{m} \mathbb{Z} \mathbf{x}_{i}
$$

The determinant of $\Lambda$, written $\operatorname{det}(\Lambda)$, is defined to be

$$
\operatorname{det}(\Lambda)=\left(\operatorname{det}_{1 \leq i, j \leq m}\left(\mathbf{X}_{i} * \mathbf{X}_{j}\right)\right)^{1 / 2},
$$

where $\mathbf{X} * \mathbf{Y}$ denotes the inner product in $\mathbb{E}$ of $\mathbf{X}$ and $\mathbf{Y}$. By convention, $\operatorname{det}(\{\mathbf{0}\})=1$.

For a subspace $S \subset K^{n}$, we write $I(S)$ for the set $S \cap \mathcal{O}_{K}^{n}$, the integral points of $S$. 
Theorem 2. If $S$ is a d-dimensional subspace of $K^{n}$, where $1 \leq d \leq n$, then $\rho(I(S))$ is a dא-dimensional lattice in $\mathbb{E}^{n \kappa}$ with

$$
H(S)=\sqrt{|\delta|}^{-d} \operatorname{det}(\rho(I(S))) .
$$

(Recall that $\delta$ is the discriminant of $K$.)

This is basically [11, Theorem 1], where a slightly different embedding is used. The proofs are entirely similar. Theorem 2 is the tool needed to compute the height of subspaces using a geometric object which is more easily studied than a point on some variety. Of course, everything we do will have an associated meaning in the context of Grassmann varieties. But the insight is from the setting of lattices in a Euclidean space, not from the variety.

We wish to prove Theorem 1 inductively, which means we will need a way to build up $d$-dimensional subspaces from smaller pieces. If $S$ is a $d$-dimensional subspace of the $(d+1)$-dimensional space $S^{+}$, then $S^{+}=S \oplus K \boldsymbol{\alpha}$, where $\alpha \in K \backslash S$. The lattice $\rho\left(I\left(S^{+}\right)\right)$will consist of the lattice $\rho(I(S))$ and a piece coming from $\alpha$. In particular, the height of $S^{+}$will be the product of the height of $S$ and a term depending on $\alpha$. We will determine this term explicitly.

For $S$ a subspace of $K^{n}, S^{(i)}=\left\{\alpha^{(i)}: \alpha \in S\right\}$ will be a subspace of

$$
\left(K^{(i)}\right)^{n} \subset \begin{cases}\mathbb{R}^{n} & \text { if } 1 \leq i \leq r_{1}, \\ \mathbb{C}^{n} & \text { if } r_{1}<i \leq \kappa .\end{cases}
$$

For a subspace $V \in \mathbb{R}^{n}$, let $V^{\perp}$ be its orthogonal complement. Similarly, for $V$ a subspace of $\mathbb{C}^{n}$, let $V^{\perp}$ be the orthogonal complement:

$$
V^{\perp}=\left\{\mathbf{x} \in \mathbb{C}^{n}: \mathbf{x} \cdot \overline{\mathbf{y}}=0 \text { for all } \mathbf{y} \in V\right\} .
$$

Let $\pi^{(i)}$ be the orthogonal projection from $\mathbb{R}^{n}$ or $\mathbb{C}^{n}$ onto $\left(S^{(i)}\right)^{\perp}$ when $1 \leq$ $i \leq r_{1}$ or $r_{1}<i \leq \kappa$, respectively. Define

$$
\pi=\pi^{(1)} \times \pi^{(2)} \times \cdots \times \pi^{(\kappa)},
$$

so that

$$
\pi(\mathbf{X})=\left(\pi^{(1)}\left(\mathbf{x}_{1}\right), \ldots, \pi^{(\kappa)}\left(\mathbf{x}_{\kappa}\right)\right)
$$

for all $\mathbf{X}=\left(\mathbf{x}_{1}, \ldots, \mathbf{x}_{\kappa}\right) \in \mathbb{E}^{n \kappa}$. Note that $\pi \circ \rho$ is linear on $K^{n}$ and vanishes only on $S$. We remark that $\left(S^{(i)}\right)^{\perp}$ is not necessarily defined over $K^{(i)}$. When we write $\pi$ we assume the subspace, $S$, is given.

Let $\tilde{\boldsymbol{\alpha}}$ be a nonzero element of the factor space $K^{n} / S$. We define

$$
\mathfrak{I}(\tilde{\boldsymbol{\alpha}})=\left\{a \in K: a \boldsymbol{\alpha} \in S+\mathfrak{D}_{K}^{n}\right\} .
$$

Note that the definition of $\mathfrak{I}(\tilde{\boldsymbol{\alpha}})$ indeed only depends on the class, $\tilde{\boldsymbol{\alpha}}$, of $\boldsymbol{\alpha}$. Clearly, $\mathfrak{I}(\tilde{\boldsymbol{\alpha}})$ is a fractional ideal. We define the height of $\tilde{\boldsymbol{\alpha}} \in K^{n} / S$ to be

$$
H(\tilde{\boldsymbol{\alpha}})=N(\mathfrak{I}(\tilde{\boldsymbol{\alpha}})) \prod_{i=1}^{\kappa}\left\|\pi^{(i)}\left(\boldsymbol{\alpha}^{(i)}\right)\right\|,
$$

where $\|\cdot\|$ denotes the usual norm on $\mathbb{R}^{n}$ if $1 \leq i \leq r_{1}$, or on $\mathbb{C}^{n}$ if $r_{1}<i \leq \kappa$. Now $\mathfrak{I}(a \tilde{\boldsymbol{\alpha}})=(1 / a) \mathfrak{I}(\tilde{\boldsymbol{\alpha}})$ for any $a \in K^{*}$, where $(1 / a)$ denotes the principal ideal generated by $1 / a$. Also

$$
\prod_{i=1}^{\kappa}\left\|\pi^{(i)}\left(a \boldsymbol{\alpha}^{(i)}\right)\right\|=\prod_{i=1}^{\kappa}\left|a^{(i)}\right| \cdot \prod_{i=1}^{\kappa}\left\|\pi^{(i)}\left(\boldsymbol{\alpha}^{(i)}\right)\right\|=N(a) \prod_{i=1}^{\kappa}\left\|\pi^{(i)}\left(\boldsymbol{\alpha}^{(i)}\right)\right\| .
$$


Hence, we may define the height of the one-dimensional subspace $K \tilde{\boldsymbol{\alpha}} \subset K^{n} / S$ as $H(K \tilde{\boldsymbol{\alpha}})=H(\tilde{\boldsymbol{\alpha}})$. Note that this skewed height reduces to the usual height when $S=\{\mathbf{0}\}$.

Theorem 3. Let $S$ be a d-dimensional subspace of $K^{n}$, where $0 \leq d<n$, and let $\tilde{\boldsymbol{\alpha}}$ be a nonzero element of the factor space $K^{n} / S$. Set $S^{+}=S \oplus K \boldsymbol{\alpha}$. Then

$$
H\left(S^{+}\right)=H(S) H(K \tilde{\boldsymbol{\alpha}}) .
$$

Proof. We will use Theorem 2. After multiplying by a suitable constant, we may assume $\mathfrak{I}(\tilde{\boldsymbol{\alpha}})$ is an integral ideal. Let $a_{1}, a_{2}, \ldots, a_{\kappa}$ be a $\mathbb{Z}$-basis for $\mathfrak{A}=\mathfrak{I}(\tilde{\boldsymbol{\alpha}})$. For each $i, 1 \leq i \leq \kappa$, there is a $\boldsymbol{\beta}_{i} \in S$ with

$$
\boldsymbol{\beta}_{i}+a_{i} \boldsymbol{\alpha} \in \mathfrak{O}_{K}^{n},
$$

by the definition of $\mathfrak{I}(\tilde{\boldsymbol{\alpha}})$. Each integral point in $S^{+}$will then be the sum of an integral point in $S$ and rational integral multiples of the points $\boldsymbol{\beta}_{i}+a_{i} \boldsymbol{\alpha}$. Thus,

$$
\rho\left(I\left(S^{+}\right)\right)=\rho(I(S)) \oplus \bigoplus_{i=1}^{\kappa} \mathbb{Z} \rho\left(\boldsymbol{\beta}_{i}+a_{i} \boldsymbol{\alpha}\right) .
$$

Now for $\mathbf{x} \in S$ and $\mathbf{y} \in K^{n}$, we have $\rho(\mathbf{x})$ and $\pi \circ \rho(\mathbf{y})$ are orthogonal. Using the linearity of $\pi \circ \rho$, and since $\pi \circ \rho$ vanishes on $S$, we obtain

$$
\operatorname{det}\left(\rho\left(I\left(S^{+}\right)\right)\right)=\operatorname{det}(\rho(I(S))) \cdot \operatorname{det}\left(\bigoplus_{i=1}^{\kappa} \mathbb{Z} \pi \circ \rho\left(a_{i} \boldsymbol{\alpha}\right)\right) .
$$

Since $\mathfrak{A}$ is integral, $N(\mathfrak{A})$ is the index of $\mathfrak{A}$ in $\mathfrak{O}_{K}$. Thus, as $\mathfrak{O}_{K}$-modules, $\mathfrak{A} \boldsymbol{\alpha}$ has index $N(\mathfrak{A})$ in $\mathfrak{D}_{K} \boldsymbol{\alpha}$. This gives

$$
\bigoplus_{i=1}^{\kappa} \mathbb{Z} \pi \circ \rho\left(a_{i} \boldsymbol{\alpha}\right)=\pi \circ \rho(\mathfrak{A} \boldsymbol{\alpha})
$$

has index $N(\mathfrak{A})$ in

$$
\bigoplus_{i=1}^{\kappa} \mathbb{Z} \pi \circ \rho\left(b_{i} \boldsymbol{\alpha}\right)=\pi \circ \rho\left(\mathcal{D}_{K} \boldsymbol{\alpha}\right),
$$

where $b_{1}, b_{2}, \ldots, b_{\kappa}$ is a $\mathbb{Z}$-basis for $\mathfrak{O}_{K}$.

We have

$$
\left(\operatorname{det}\left(\bigoplus_{i=1}^{\kappa} \mathbb{Z} \pi \circ \rho\left(b_{i} \boldsymbol{\alpha}\right)\right)\right)^{2}=\operatorname{det}_{1 \leq i, j \leq \kappa}\left(\boldsymbol{\alpha}_{i} \cdot \boldsymbol{\alpha}_{j}\right),
$$

where $\boldsymbol{\alpha}_{i}$ is defined to be $\pi \circ \rho\left(b_{i} \boldsymbol{\alpha}\right)$ for $1 \leq i \leq \kappa$. From the definition of $\pi$ and $\rho$, we see that

$$
\boldsymbol{\alpha}_{i} \cdot \boldsymbol{\alpha}_{j}=\sum_{l=1}^{\kappa} b_{i}^{(l)} b_{j}^{(l)}\left\|\pi^{(l)}\left(\boldsymbol{\alpha}^{(l)}\right)\right\|^{2}
$$


so that the matrix $\left(\boldsymbol{\alpha}_{i} \cdot \boldsymbol{\alpha}_{j}\right)_{1 \leq i, j \leq \kappa}$ is the product

$$
\begin{gathered}
\left(\begin{array}{ccc}
b_{1}^{(1)}\left\|\pi^{(1)}\left(\boldsymbol{\alpha}^{(1)}\right)\right\| & \ldots & b_{1}^{(\kappa)}\left\|\pi^{(\kappa)}\left(\boldsymbol{\alpha}^{(\kappa)}\right)\right\| \\
\vdots & \ddots & \vdots \\
b_{\kappa}^{(1)}\left\|\pi^{(1)}\left(\boldsymbol{\alpha}^{(1)}\right)\right\| & \ldots & b_{\kappa}^{(\kappa)}\left\|\pi^{(\kappa)}\left(\boldsymbol{\alpha}^{(\kappa)}\right)\right\|
\end{array}\right) \\
\quad\left(\begin{array}{ccc}
b_{1}^{(1)}\left\|\pi^{(1)}\left(\boldsymbol{\alpha}^{(1)}\right)\right\| & \ldots & b_{\kappa}^{(1)}\left\|\pi^{(1)}\left(\boldsymbol{\alpha}^{(1)}\right)\right\| \\
\vdots & \ddots & \vdots \\
b_{1}^{(\kappa)}\left\|\pi^{(\kappa)}\left(\boldsymbol{\alpha}^{(\kappa)}\right)\right\| & \ldots & b_{\kappa}^{(\kappa)}\left\|\pi^{(\kappa)}\left(\boldsymbol{\alpha}^{(\kappa)}\right)\right\|
\end{array}\right)
\end{gathered}
$$

and the determinant

$$
\underset{1 \leq i, j \leq \kappa}{\operatorname{det}}\left(\boldsymbol{\alpha}_{i} \cdot \boldsymbol{\alpha}_{j}\right)=\delta \prod_{i=1}^{\kappa}\left\|\pi^{(i)}\left(\boldsymbol{\alpha}^{(i)}\right)\right\|^{2} .
$$

We thus have

$$
\operatorname{det}\left(\bigoplus_{i=1}^{\kappa} \mathbb{Z} \pi \circ \rho\left(b_{i} \boldsymbol{\alpha}\right)\right)=|\delta|^{1 / 2} \prod_{i=1}^{\kappa}\left\|\pi^{(i)}\left(\boldsymbol{\alpha}^{(i)}\right)\right\| .
$$

Theorem 3 now follows from (4), (5), and Theorem 2.

In what follows, we will also need the following result.

Lemma 1. Let $\mathfrak{M}$ be an $\mathfrak{D}_{K}$-module in $K^{n}$ spanning a subspace of dimension $d$ and let $\mathfrak{A}$ be any ideal. If we let $\mathfrak{A M}$ denote the set of finite sums

$$
\left\{\sum a_{i} m_{i}: a_{i} \in \mathfrak{A} \text { and } m_{i} \in \mathfrak{M}\right\}
$$

then we have

$$
\operatorname{det}(\rho(\mathfrak{A M}))=N(\mathfrak{A})^{d} \operatorname{det}(\rho(\mathfrak{M})) .
$$

Proof. First suppose $\mathfrak{A}$ is a prime ideal. We will localize. Let $S=\mathfrak{O}_{K} \backslash \mathfrak{A}$ and $\mathfrak{O}_{\mathfrak{A}}=S^{-1} \mathfrak{O}_{K}$. Then $\mathfrak{M}_{\mathfrak{A}}=S^{-1} \mathfrak{M}$ and $S^{-1} \mathfrak{A} \mathfrak{M}_{\mathfrak{A}}$ are both $\mathfrak{O}_{\mathfrak{A}}$-modules with

$$
\left[\mathfrak{M}_{\mathfrak{A}}: S^{-1} \mathfrak{A} \mathfrak{M}_{\mathfrak{A}}\right]=[\mathfrak{M}: \mathfrak{A M}] \text { and }\left[\mathfrak{O}_{\mathfrak{A}}: S^{-1} \mathfrak{A}\right]=\left[\mathfrak{O}_{K}: \mathfrak{A}\right]=N(\mathfrak{A})
$$

(see, for example, [1, Chapter 13]). The purpose of localizing is that $\mathfrak{O}_{\mathfrak{A}}$ is a principal ideal domain, so that $\mathfrak{M}_{\mathfrak{A}}$ is a free $\mathfrak{O}_{\mathfrak{A}}$-module of rank $d$. We thus have

$$
[\mathfrak{M}: \mathfrak{A M}]=\left[\mathfrak{M}_{\mathfrak{A}}: S^{-1} \mathfrak{A M}_{\mathfrak{A}}\right]=\left[\mathfrak{O}_{\mathfrak{A}}: S^{-1} \mathfrak{A}\right]^{d}=N(\mathfrak{A})^{d} .
$$

Since $\rho$ is one-to-one on $K^{n}$, the result holds for $\mathfrak{A}$ prime, and hence for any integral $\mathfrak{A}$.

In general, let $\mathfrak{A}=\mathfrak{B}^{-1} \mathfrak{C}$, where $\mathfrak{B}$ and $\mathfrak{C}$ are both integral ideals. By what we have shown,

$$
N(\mathfrak{B})^{d} \operatorname{det}(\rho(\mathfrak{A M}))=\operatorname{det}(\rho(\mathfrak{C M}))=N(\mathfrak{C})^{d} \operatorname{det}(\rho(\mathfrak{M})),
$$

which proves the lemma in general.

\section{The Main Term and the Main Error Term}

We will prove Theorem 1 by induction on $n$. We will need the following result. 
Theorem 4. For $1 \leq d<n$, we have $M(n, d, B) \ll B^{n}$, where the constant implicit in the notation depends only on $K$ and $n$.

This is one half of [11, Theorem 3]. This will also be a corollary of Lemma 15 below.

We think of $K^{n-1}$ as being embedded in $K^{n}$ :

$$
K^{n-1}=\left\{\boldsymbol{\alpha}=\left(\alpha_{1}, \alpha_{2}, \ldots, \alpha_{n-1}, 0\right) \in K^{n}\right\} .
$$

By Theorem 4, $M(n-1, d, B)=O\left(B^{n-1}\right)$. Thus, it suffices to count $d$ dimensional subspaces $S$ of $K^{n}$ satisfying $S \not \subset K^{n-1}$. Suppose $S$ is such a subspace. We then have a unique $(d-1)$-dimensional subspace of $K^{n-1}$, namely $S^{-}=S \cap K^{n-1}$, and a one-dimensional subspace $K \tilde{\boldsymbol{\alpha}} \subset K^{n} / S^{-}$with

$$
S=S^{-} \oplus K \boldsymbol{\alpha} .
$$

Since $S \not \subset K^{n-1}$, we may choose the unique representative $\boldsymbol{\alpha}$ of the class $\tilde{\boldsymbol{\alpha}}$ with last coordinate equal to 1 . So $\alpha$ is of the type $\left(\beta_{1}, \beta_{2}, \ldots, \beta_{n-1}, 1\right)=$ $(\boldsymbol{\beta}, 1)$, say, where $\boldsymbol{\beta} \in K^{n-1}$.

Now suppose $S^{-}$is a $(d-1)$-dimensional subspace of $K^{n-1}$. Let

$$
\rho: K^{n-1} \rightarrow \mathbb{E}^{(n-1) \kappa}
$$

be defined as above, with $n-1$ in place of $n$. For $\tilde{\boldsymbol{\beta}}$ in the factor space $K^{n-1} / S^{-}$, let

$$
\mathfrak{I}(\tilde{\boldsymbol{\beta}})=\left\{a \in K: a \boldsymbol{\beta} \in S^{-}+\mathfrak{D}_{K}^{n-1}\right\},
$$

as above. Define $\mathfrak{I}^{*}(\tilde{\boldsymbol{\beta}})=\mathfrak{I}(\tilde{\boldsymbol{\beta}}) \cap \mathfrak{O}_{K}$, the largest integral ideal contained in $\mathfrak{I}(\tilde{\boldsymbol{\beta}})$.

Let $(\widetilde{\boldsymbol{\beta}, 1}) \in K^{n} / S$ be the class of $(\boldsymbol{\beta}, 1)$. We easily have

$$
\mathfrak{I}((\widetilde{\boldsymbol{\beta}, 1}))=\left\{a \in K: a(\boldsymbol{\beta}, 1) \in S+\mathfrak{O}_{K}^{n}\right\}=\mathfrak{I}^{*}(\tilde{\boldsymbol{\beta}}) .
$$

We summarize this discussion with a lemma.

Lemma 2. Let $S^{-}$be a $(d-1)$-dimensional subspace of $K^{n-1}$, where $1 \leq d<$ $n$, and $\tilde{\boldsymbol{\beta}} \in K^{n-1} / S^{-}$. Then the $d$-dimensional subspace $S \subset K^{n}$ given by $S=S^{-} \oplus K(\boldsymbol{\beta}, 1)$ has height

$$
H(S)=H\left(S^{-}\right) N\left(\mathfrak{I}^{*}(\tilde{\boldsymbol{\beta}})\right) \prod_{i=1}^{r_{1}+r_{2}}\left(\left\|\pi^{(i)}\left(\boldsymbol{\beta}^{(i)}\right)\right\|^{2}+1\right)^{e_{i} / 2},
$$

where $\pi^{(i)}$ is defined as above, with respect to $S^{-}$. There is a 1-1 correspondence between such pairs $\left(S^{-}, \tilde{\boldsymbol{\beta}}\right)$ and d-dimensional subspaces $S \subset K^{n}$ with $S \not \subset$ $K^{n-1}$, i.e., if

$$
S_{1}^{-} \oplus K\left(\boldsymbol{\beta}_{1}, 1\right)=S_{2}^{-} \oplus K\left(\boldsymbol{\beta}_{2}, 1\right),
$$

where $S_{1}^{-}, S_{2}^{-} \subset K^{n-1}$ and $\tilde{\boldsymbol{\beta}}_{i} \in K^{n-1} / S_{i}^{-}$for $i=1$ and 2 , then

$$
S_{1}^{-}=S_{2}^{-} \quad \text { and } \quad \tilde{\boldsymbol{\beta}}_{1}=\tilde{\boldsymbol{\beta}}_{2} \text {, }
$$

and every $S \subset K^{n}$ with $S \not \subset K^{n-1}$ has such a decomposition.

Proof. The only statement remaining to be proven is (6). This is an easy application of Theorem 3 , using $\mathfrak{I}^{*}(\tilde{\boldsymbol{\beta}})=\mathfrak{I}(\widetilde{\boldsymbol{\beta}, 1})$. 
It will be convenient to have a more compact notation for

$$
\prod_{i=1}^{r_{1}+r_{2}}\left(\left\|\pi^{(i)}\left(\boldsymbol{\beta}^{(i)}\right)\right\|^{2}+1\right)^{e_{i} / 2} .
$$

We will henceforth write $H_{S}(\boldsymbol{\beta}, 1)$ for this quantity.

Define the following sets:

$L(\mathfrak{A}, S, B)=\left\{\tilde{\boldsymbol{\beta}} \in K^{n-1} / S: \tilde{\boldsymbol{\beta}} \neq \tilde{\mathbf{0}}, \mathfrak{I}(\tilde{\boldsymbol{\beta}}) \supset \mathfrak{A}\right.$ and $\left.H_{S}(\boldsymbol{\beta}, 1) \leq \frac{B}{N(\mathfrak{A}) H(S)}\right\}$

and

$$
\bar{L}(\mathfrak{A}, S, B)=\left\{\tilde{\boldsymbol{\beta}} \in L(\mathfrak{A}, S, B): \mathfrak{I}^{*}(\tilde{\boldsymbol{\beta}})=\mathfrak{A}\right\},
$$

where $S$ is a $(d-1)$-dimensional subspace of $K^{n-1}$ and $\mathfrak{A}$ is any fractional ideal. Note that $\bar{L}(\mathfrak{A}, S, B)$ is empty if $\mathfrak{A}$ is not integral, since $\mathfrak{I}^{*}(\tilde{\boldsymbol{\beta}})$ is always integral. Denote the cardinalities of $L(\mathfrak{A}, S, B)$ and $\bar{L}(\mathfrak{A}, S, B)$ by $\lambda(\mathfrak{A}, S, B)$ and $\bar{\lambda}(\mathfrak{A}, S, B)$, respectively. We will compute $L(\mathfrak{A}, S, B)$ and recapture $\bar{L}(\mathfrak{A}, S, B)$ with an inversion.

Let $\mu$ be the Möbius function on ideals: $\mu\left(\mathfrak{O}_{K}\right)=1, \mu\left(\mathfrak{P}^{v}\right)=-1$ if $\mathfrak{P}$ is a prime ideal and $v=1$ and $=0$ otherwise, and $\mu(\mathfrak{A B})=\mu(\mathfrak{A}) \mu(\mathfrak{B})$ if $\mathfrak{A}$ and $\mathfrak{B}$ are relatively prime.

One easily verifies that, as in the case for the rational integers (see [5]),

$$
\sum_{\mathfrak{B} \mid \mathfrak{I}} \mu(\mathfrak{B})= \begin{cases}1 & \text { if } \mathfrak{I}=\mathfrak{O}_{K} \\ 0 & \text { otherwise }\end{cases}
$$

Lemma 3. For $\mathfrak{A}$ integral,

$$
\bar{\lambda}(\mathfrak{A}, S, B)=\sum_{\mathfrak{C} \mid \mathfrak{A}} \mu(\mathfrak{C}) \lambda\left(\mathfrak{A} \mathfrak{C}^{-1}, S, B / N(\mathfrak{C})\right) .
$$

Proof. For $\mathfrak{A}$ integral,

$$
\lambda(\mathfrak{A}, S, B)=\sum_{\mathfrak{C} \mid \mathfrak{A}} \bar{\lambda}\left(\mathfrak{C}, S, \frac{B N(\mathfrak{C})}{N(\mathfrak{A})}\right) .
$$

Setting $\mathfrak{B}=\mathfrak{A} \mathfrak{C}^{-1}$ gives

$$
\lambda(\mathfrak{A}, S, B)=\sum_{\mathfrak{B} \mid \mathfrak{A}} \bar{\lambda}\left(\mathfrak{A B}^{-1}, S, B / N(\mathfrak{B})\right) .
$$

Thus,

$$
\begin{aligned}
\sum_{\mathfrak{C} \mid \mathfrak{A}} \mu(\mathfrak{C}) \lambda\left(\mathfrak{A} \mathfrak{C}^{-1}, S, \frac{B}{N(\mathfrak{C})}\right) \\
=\sum_{\mathfrak{C} \mid \mathfrak{A}} \sum_{\mathfrak{B} \mid \mathfrak{A} \mathfrak{C}-1} \mu(\mathfrak{C}) \bar{\lambda}\left(\mathfrak{A}(\mathfrak{C} \mathfrak{B})^{-1}, S, \frac{B}{N(\mathfrak{C}) N(\mathfrak{B})}\right) \\
=\sum_{\mathfrak{I} \mid \mathfrak{A}} \bar{\lambda}\left(\mathfrak{A I}^{-1}, S, \frac{B}{N(\mathfrak{I})}\right) \sum_{\mathfrak{C} \mid \mathfrak{I}} \mu(\mathfrak{C}) \\
=\bar{\lambda}(\mathfrak{A}, S, B) \quad(\text { by }(7)),
\end{aligned}
$$

where $\mathfrak{I}=\mathfrak{B C}$. 
Lemma 4. For $1 \leq d<n$,

$$
M(n, d, B)=\sum_{\mathfrak{A}} \sum_{S} \sum_{\mathfrak{C} \mid \mathfrak{A}} \mu(\mathfrak{C}) \lambda\left(\mathfrak{A} \mathfrak{C}^{-1}, S, B / N(\mathfrak{C})\right)+O\left(B^{n-1}\right),
$$

where the first sum is over integral ideals $\mathfrak{A}$ satisfying $N(\mathfrak{A}) \leq B$, the second sum is over $(d-1)$-dimensional subspaces $S \subset K^{n-1}$ satisfying $H(S) \leq$ $B / N(\mathfrak{A})$, and the constant implicit in the $O$ notation depends only on $K$ and $n$.

Proof. Note that $H_{S}(\boldsymbol{\beta}, 1) \geq 1$. Thus,

$$
\bar{L}(\mathfrak{A}, S, B) \subset L(\mathfrak{A}, S, B)=\varnothing \quad \text { if } \frac{B}{N(\mathfrak{A}) H(S)}<1 .
$$

By [11, Lemma 8], $H(S) \geq 1$, so both sets are empty if $N(\mathfrak{A})>B$. By Lemma 2 and Theorem 4 ,

$$
M(n, d, B)=\sum_{\mathfrak{A}} \sum_{S} \bar{\lambda}(\mathfrak{A}, S, B)+O\left(B^{n-1}\right) .
$$

The lemma follows from Lemma 3.

We estimate $M(n, d, B)$ by estimating $\lambda\left(\mathfrak{A} \mathfrak{C}^{-1}, S, B / N(\mathfrak{C})\right)$ and using Lemma 4. We transform this into estimating the number of lattice points in a domain.

For the remainder of this paper, the constant implicit in the $\ll$ notation will depend only on $n$ and $K$, unless specifically stated otherwise. Also, we will use the notation $\mathfrak{B}^{n-1}$ for the subset of $K^{n-1}$,

$$
\underbrace{\mathfrak{B} \times \mathfrak{B} \times \cdots \times \mathfrak{B}}_{n-1 \text { times }},
$$

where $\mathfrak{B}$ is any fractional ideal.

We map $\mathbb{E}^{(n-1) \kappa}$ into $\mathbb{R}^{(n-1) \kappa}$ by a linear transformation $T: \mathbb{E}^{(n-1) \kappa} \rightarrow$ $\mathbb{R}^{(n-1) \kappa}$, defined by

$$
\begin{aligned}
T(\mathbf{X}) & =T\left(\left(\mathbf{x}_{1}, \mathbf{x}_{2}, \ldots, \mathbf{x}_{\kappa}\right)\right) \\
& =\left(\mathbf{x}_{1}, \mathbf{x}_{2}, \ldots, \mathbf{x}_{r_{1}}, \mathbf{x}_{r_{1}+1}^{\prime}, \mathbf{x}_{r_{1}+2}^{\prime}, \ldots, \mathbf{x}_{r_{1}+r_{2}}^{\prime}\right),
\end{aligned}
$$

where, for $\mathbf{x}_{i}=\left(x_{i 1}, x_{i 2}, \ldots, x_{i(n-1)}\right)$ and $i=r_{1}+1, r_{1}+2, \ldots, r_{1}+r_{2}$, we define

$$
\mathbf{x}_{i}^{\prime}=\left(\operatorname{Re}\left(x_{i 1}\right), \operatorname{Im}\left(x_{i 1}\right), \ldots, \operatorname{Re}\left(x_{i(n-1)}\right), \operatorname{Im}\left(x_{i(n-1)}\right)\right) .
$$

One sees that the determinant of $T$ is $2^{-r_{2}(n-1)}$. For $\mathbf{Y} \in \mathbb{R}^{(n-1) \kappa}$, we write

$$
\mathbf{Y}=\left(\mathbf{y}_{1}, \mathbf{y}_{2}, \ldots, \mathbf{y}_{r_{1}+r_{2}}\right) \text {, }
$$

where

$$
\mathbf{y}_{i} \in \begin{cases}\mathbb{R}^{n-1} & \text { for } 1 \leq i \leq r_{1} \\ \mathbb{R}^{2(n-1)} & \text { for } r_{1}+1 \leq i \leq r_{1}+r_{2}\end{cases}
$$

Now let $\Lambda$ be an l-dimensional lattice in $\mathbb{R}^{k}$ spanning a subspace $V$. Let $\lambda_{1} \leq \lambda_{2} \leq \cdots \leq \lambda_{l}$ be the successive minima of $\Lambda$ with respect to the unit ball in $V$. Pick linearly independent points $\mathbf{y}_{i} \in \Lambda$ (the choice is not necessarily unique) satisfying $\left|\mathbf{y}_{i}\right|=\lambda_{i}$ for $i=1,2, \ldots, l$. Define

$$
\Lambda^{-i}=\Lambda \cap \bigoplus_{j=1}^{l-i} \mathbb{R} \mathbf{y}_{j} \quad \text { for } i=1,2, \ldots, l-1
$$


and $\Lambda^{-l}=\{\boldsymbol{0}\}$. Minkowski's second convex bodies theorem [2, Chapter VIII] asserts that

$$
\frac{2^{l}}{l !} \operatorname{det}(\Lambda) \leq \lambda_{1} \lambda_{2} \cdots \lambda_{l} V(l) \leq 2^{l} \operatorname{det}(\Lambda)
$$

Since the successive minima of $\Lambda^{-i}$ are, by construction, $\lambda_{1} \leq \lambda_{2} \leq \cdots \leq \lambda_{l-i}$ for $i<l$, we have $\operatorname{det}\left(\Lambda^{-i}\right)$ is minimal among sublattices of $\Lambda$ of dimension $l-i$.

We have that $\lambda\left(\mathfrak{A C} \mathfrak{C}^{-1}, S, B / N(\mathfrak{C})\right)$ is precisely the number of nonzero lattice points $\mathbf{X} \in \pi \circ \rho\left(\left(\mathfrak{C A}^{-1}\right)^{n-1}\right) \subset \mathbb{E}^{(n-1) \kappa}$ in the domain

$$
\left\{\mathbf{X} \in \mathbb{E}^{(n-1) \kappa}: \mathbf{X} \in V \text { and } \prod_{i=1}^{\kappa}\left(\left\|\mathbf{x}_{i}\right\|^{2}+1\right)^{1 / 2} \leq \frac{B}{H(S) N(\mathfrak{A})}\right\},
$$

where $V$ is the subspace of $\mathbb{E}^{(n-1) \kappa}$ spanned by $\pi \circ \rho\left(\left(\mathfrak{C} \mathfrak{A}^{-1}\right)^{n-1}\right)$. Equivalently, if we denote by $T^{\prime}$ the composition $T \circ \pi \circ \rho$, then $\lambda\left(\mathfrak{A} \mathfrak{C}^{-1}, S, B / N(\mathfrak{C})\right)$ is the number of nonzero lattice points $Y \in T^{\prime}\left(\left(\mathfrak{C} \mathfrak{A}^{-1}\right)^{n-1}\right)$ in the domain

$$
\left\{\mathbf{Y} \in \mathbb{R}^{(n-1) \kappa}: \mathbf{Y} \in T(V) \text { and } \prod_{i=1}^{r_{1}+r_{2}}\left(\left\|\mathbf{y}_{i}\right\|^{2}+1\right)^{e_{i} / 2} \leq \frac{B}{H(S) N(\mathfrak{A})}\right\} .
$$

For $x \in(0, B / N(\mathfrak{A})]$ and $0<m \leq(n-d) \kappa$, let $V(\mathfrak{A}, x, B, m)$ denote the sum of the $m$-dimensional volumes of the projections of the domain

$$
D(x)=\left\{\mathbf{Y} \in \mathbb{R}^{(n-d) \kappa}: \prod_{i=1}^{r_{1}+r_{2}}\left(\left\|\mathbf{y}_{i}\right\|^{2}+1\right)^{e_{i} / 2} \leq \frac{B}{x N(\mathfrak{A})}\right\}
$$

on the various coordinate spaces obtained by equating $(n-d) \kappa-m$ of the coordinates to zero. Let $V(\mathfrak{A}, x, B, 0)=1$.

We define the Main Term to be

$$
\sum_{\mathfrak{A}} \sum_{\mathfrak{C} \mid \mathfrak{A}} \sum_{S} \mu(\mathfrak{C}) \frac{V(\mathfrak{A}, H(S), B,(n-d) \kappa)}{\operatorname{det}\left(T^{\prime}\left(\left(\mathfrak{C} \mathfrak{A}^{-1}\right)^{n-1}\right)\right)}
$$

and the Main Error Term to be

$$
\sum_{\mathfrak{A}} \sum_{\mathfrak{C} \mid \mathfrak{A}} \sum_{S} \sum_{m=0}^{(n-d) \kappa-1} \frac{V(\mathfrak{A}, H(S), B, m)}{\operatorname{det}\left[\left(T^{\prime}\left(\left(\mathfrak{C} \mathfrak{A}^{-1}\right)^{n-1}\right)\right)^{-[(n-d) \kappa-m]}\right]},
$$

where in both cases the first sum is over integral ideals $\mathfrak{A}$ satisfying $N(\mathfrak{A}) \leq B$ and the third sum is over $(d-1)$-dimensional subspaces $S \subset K^{n-1}$ satisfying $H(S) \leq B / N(\mathfrak{A})$.

Lemma 5. For $1 \leq d<n$,

$$
\mid M(n, d, B)-\text { Main Term } \mid \ll \text { Main Error Term }+O\left(B^{n-1}\right) \text {. }
$$

Proof. Fix a $(d-1)$-dimensional subspace $S \subset K^{n-1}$ and integral ideals $\mathfrak{A}$ and $\mathfrak{C}$. After applying a unitary transformation, $\tau$, of $\mathbb{R}^{(n-1) \kappa}$, we have $\lambda\left(\mathfrak{C} \mathfrak{A}^{-1}, S, B / N(\mathfrak{C})\right)$ is the number of nonzero lattice points

$$
\mathbf{Y} \in \tau\left(T^{\prime}\left(\left(\mathfrak{C} \mathfrak{A}^{-1}\right)^{n-1}\right)\right)=\Lambda \subset \mathbb{R}^{(n-d) \kappa}
$$

in $D(H(S))$. 
Notice that $D(H(S))$ is a domain such that any line intersects it in a set of points which, if not empty, consists of the union of at most $(n-d) \kappa$ intervals. Also notice that if

$$
\mathbf{Y}=\left(y_{1}, y_{2}, \ldots, y_{(n-d) \kappa}\right) \in D(H(S)),
$$

then

$$
\mathbf{Y}^{\prime}=\left(y_{1}^{\prime}, y_{2}^{\prime}, \ldots, y_{(n-d) \kappa}^{\prime}\right) \in D(H(S))
$$

if $\left|y_{i}^{\prime}\right| \leq\left|y_{i}\right|$ for $1 \leq i \leq(n-d) \kappa$. Lemma 5 thus follows from [13, Theorem 2 or the appendix] and Lemma 4.

\section{Refinements in the Main ERror Term}

The terms in Lemma 5 look fairly nasty as they stand, especially the lattices. It is the goal of this section to get a more workable formulation of Lemma 5.

Let $\Lambda$ be a lattice in a euclidean space $\mathbb{E}$, with inner product $*$, spanning a subspace $V$. The polar lattice, $\Lambda^{P}$, is defined to be the set of all points $\mathbf{y} \in V$ with rational integral inner products $\mathbf{y} * \mathbf{x}$ for all $\mathbf{x} \in \Lambda$.

Lemma 6. Let $S$ be a $(d-1)$-dimensional subspace of $K^{n-1}$ and let $S^{n-d}$ be the "orthogonal complement" of $S$ in $K^{n-1}$ defined in the Introduction. Let $\mathfrak{B}$ be any fractional ideal. Then, for $\mathbf{X}=\left(\mathbf{x}_{1}, \mathbf{x}_{2}, \ldots, \mathbf{x}_{\kappa}\right) \in \pi \circ \rho\left(\mathfrak{B}^{n-1}\right)$, the complex conjugate of $\mathbf{X}$ lies in the polar lattice:

$$
\overline{\mathbf{X}}=\left(\mathbf{x}_{1}, \mathbf{x}_{2}, \ldots, \mathbf{x}_{r_{1}}, \overline{\mathbf{x}_{r_{1}+1}}, \ldots, \overline{\mathbf{x}_{\kappa}}\right) \in\left(\rho\left(\mathfrak{B}^{-1} I\left(S^{n-d}\right)\right)\right)^{P} .
$$

Proof. We have $\mathbf{X}=\mathbf{Y}+\mathbf{Z}$, where $\mathbf{Y}=\left(\mathbf{y}^{(1)}, \mathbf{y}^{(2)}, \ldots, \mathbf{y}^{(\kappa)}\right) \in \rho\left(\mathfrak{B}^{n-1}\right)$ and $\mathbf{Z}=\left(\mathbf{z}_{1}, \mathbf{z}_{2}, \ldots, \mathbf{z}_{\kappa}\right)$ satisfies $\mathbf{z}_{i} \in S^{(i)}$ for $i=1,2, \ldots, \kappa$. Let

$$
\mathbf{W}=\left(\mathbf{w}^{(1)}, \mathbf{w}^{(2)}, \ldots, \mathbf{w}^{(\kappa)}\right) \in \rho\left(\mathfrak{B}^{-1} I\left(S^{n-d}\right)\right) .
$$

Then (recall the definition of inner product in $\mathbb{E}^{(n-d) \kappa}$ )

$$
\overline{\mathbf{X}} \cdot \mathbf{W}=\sum_{i=1}^{\kappa} \mathbf{x}_{i} \cdot \mathbf{w}_{i}=\sum_{i=1}^{\kappa} \mathbf{y}^{(i)} \cdot \mathbf{w}^{(i)}+\sum_{i=1}^{\kappa} \mathbf{z}_{i} \cdot \mathbf{w}^{(i)}=\sum_{i=1}^{\kappa}(\mathbf{y} \cdot \mathbf{w})^{(i)},
$$

since $\mathbf{z}_{i} \cdot \mathbf{w}^{(i)}=0^{(i)}=0$ for all $i=1,2, \ldots, \kappa$. But since the components of $\mathbf{y}$ are in $\mathfrak{B}$ and the components of $\mathbf{w}$ are in $\mathfrak{B}^{-1}$, the product $\mathbf{y} \cdot \mathbf{w}$ will be integral. The last sum is the trace of an integer, hence a rational integer, and the lemma is proved.

We may replace the $T^{\prime}\left(\left(\mathfrak{C} \mathfrak{A}^{-1}\right)^{n-1}\right)$ occurring in the Main Error Term with $\pi \circ \rho\left(\left(\mathfrak{C A}^{-1}\right)^{n-1}\right)$, since $T$ is a linear transformation which only introduces a constant multiple depending on $K$ and $n$ in the determinant of $\pi \circ \rho\left(\left(\mathfrak{C} \mathfrak{A}^{-1}\right)^{n-1}\right)$. By Lemma 6, we may replace this lattice with $\left(\rho\left(\mathfrak{A C} \mathfrak{C}^{-1} I\left(S^{n-d}\right)\right)\right)^{P}$. One may well ask if we are "giving up" too much by doing this. We will show here that we are not: making this change will only introduce another constant multiple depending on $K$ and $n$, and, in fact, the two lattices are the same when $K=\mathbb{Q}$.

Every vector in $\rho\left(\left(\mathfrak{C} \mathfrak{A}^{-1}\right)^{n-1}\right)$ will either be in $\rho\left(\mathfrak{C} \mathfrak{A}^{-1} I(S)\right)$ or will be the sum of a vector in $\rho(S)$ and a vector in $\pi \circ \rho\left(\left(\mathfrak{C} \mathfrak{A}^{-1}\right)^{n-1}\right)$. We thus have, by 
Theorem 2 and Lemma 1,

$$
\begin{aligned}
\operatorname{det}\left(\pi \circ \rho\left(\left(\mathfrak{C} \mathfrak{A}^{-1}\right)^{n-1}\right)\right) & =\frac{\operatorname{det}\left(\rho\left(\left(\mathfrak{C} \mathfrak{A}^{-1}\right)^{n-1}\right)\right)}{\operatorname{det}\left(\rho\left(\mathfrak{C} \mathfrak{A}^{-1} I(S)\right)\right)} \\
& =\frac{N\left(\mathfrak{C} \mathfrak{A}^{-1}\right)^{n-1} \sqrt{|\delta|}^{n-1}}{N\left(\mathfrak{C} \mathfrak{A}^{-1}\right)^{d-1} \sqrt{|\delta|}^{d-1} H(S)} \\
& =N\left(\mathfrak{C} \mathfrak{A}^{-1}\right)^{n-d} \sqrt{|\delta|}^{n-d} H(S)^{-1} .
\end{aligned}
$$

It is easily shown (see [2, Chapter 1, Lemma 5]) that the determinant of the polar lattice is the reciprocal of the determinant of the original lattice. Therefore, by Theorem 2 and Lemma 1,

$$
\begin{aligned}
& \operatorname{det}\left(\rho\left(\mathfrak{A C} \mathfrak{C}^{-1} I\left(S^{n-d}\right)\right)^{P}\right)=\left[\operatorname{det}\left(\rho\left(\mathfrak{A C} \mathfrak{C}^{-1} I\left(S^{n-d}\right)\right)\right)\right]^{-1} \\
& =N\left(\mathfrak{C} \mathfrak{A}^{-1}\right)^{n-d} \sqrt{|\delta|}^{d-n} H\left(S^{n-d}\right)^{-1} \\
& =\sqrt{|\delta|}^{2(d-n)} \operatorname{det}\left(\pi \circ \rho\left(\mathfrak{C} \mathfrak{A}^{-1} I(S)\right)\right),
\end{aligned}
$$

since $H\left(S^{n-d}\right)=H(S)$.

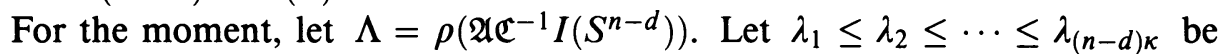
the successive minima of $\Lambda$ and $\mu_{1} \leq \mu_{2} \leq \cdots \leq \mu_{(n-d) \kappa}$ be the successive minima of $\Lambda^{P}$ with respect to the unit ball in the space spanned by $\Lambda$. By Mahler [9] (Theorem VI of Chapter VIII in [2]),

$$
c \leq \lambda_{i} \mu_{(n-d) \kappa-i+1} \leq c^{\prime} \quad \text { for } i=1,2, \ldots,(n-d) \kappa,
$$

where $c$ and $c^{\prime}$ are constants depending only on $(n-d) \kappa$.

Using this fact together with Minkowski's theorem yields

$$
\begin{aligned}
{\left[\operatorname{det}\left(\left(\Lambda^{P}\right)^{-i}\right)\right]^{-1} } & \ll \prod_{j=1}^{(n-d) \kappa-i} \mu_{j}^{-1} \ll \prod_{j=i+1}^{(n-d) \kappa} \lambda_{j} \\
& =\frac{\prod_{j=1}^{(n-d) \kappa} \lambda_{j}}{\prod_{j=1}^{i} \lambda_{j}} \ll \frac{\operatorname{det}(\Lambda)}{\operatorname{det}\left(\Lambda^{-[(n-d) \kappa-i]}\right)}
\end{aligned}
$$

for $i=1,2, \ldots,(n-d) \kappa$. We summarize our discussion up to this point.

Lemma 7. The Main Error Term is

$$
\ll \sum_{\mathfrak{A}} \sum_{\mathfrak{C} \mid \mathfrak{A}} \sum_{S} \sum_{m=0}^{(n-d) \kappa-1} V(\mathfrak{A}, H(S), B, m) \frac{\operatorname{det}\left(\rho\left(\mathfrak{A C} \mathfrak{C}^{-1} I(S)\right)\right)}{\operatorname{det}\left(\rho\left(\mathfrak{A C} \mathfrak{C}^{-1} I(S)\right)^{-m}\right)},
$$

where the first sum is over integral ideals $\mathfrak{A}$ satisfying $N(\mathfrak{A}) \leq B$ and the third sum is over $(n-d)$-dimensional subspaces $S \subset K^{n-1}$ satisfying $H(S) \leq$ $B / N(\mathfrak{A})$.

We now determine the growth of the successive minima of $\rho\left(\mathfrak{A C} \mathfrak{C}^{-1} I(S)\right)$.

Lemma 8. Let $\alpha \in \mathfrak{B} I(S)$, where $S$ is any subspace of $K^{n}$ and $\mathfrak{B}$ is any fractional ideal. Suppose $\boldsymbol{\alpha} \neq \mathbf{0}$ and let $\lambda=\|\rho(\boldsymbol{\alpha})\|$. Then there are $\alpha_{1}, \alpha_{2}, \ldots$, $\boldsymbol{\alpha}_{\kappa} \in \mathfrak{B I}(S)$, linearly independent over $\mathbb{Q}$, satisfying $\left\|\rho\left(\boldsymbol{\alpha}_{i}\right)\right\| \ll \lambda$ for $1 \leq i \leq$ $\kappa$. In particular, if $a \in K^{*}$ we have

$$
\|\rho(a \boldsymbol{\alpha})\| \leq c(a) \lambda,
$$

where $c(a)$ is a constant depending only on $a$. 
Proof. We have

$$
\|\rho(\boldsymbol{\alpha})\|=\left(\sum_{j=1}^{\kappa}\left\|\boldsymbol{\alpha}^{(j)}\right\|^{2}\right)^{1 / 2}
$$

Letting $c(a)=\max _{1 \leq j \leq \kappa}\left|a^{(j)}\right|$ gives (9).

Now choose a $\mathbb{Z}$-basis $\beta_{1}, \beta_{2}, \ldots, \beta_{\kappa}$ of $\mathfrak{O}_{K}$. We let $\alpha_{i}=\beta_{i} \alpha$. Then $\boldsymbol{\alpha}_{i} \in \mathfrak{B} I(S)$ and $\left\|\rho\left(\boldsymbol{\alpha}_{i}\right)\right\| \ll\|\rho(\boldsymbol{\alpha})\|$ for $1 \leq i \leq \kappa$. Since the $\beta_{i}$ 's are a basis for $K$ over $\mathbb{Q}, \boldsymbol{\alpha}_{i}, \boldsymbol{\alpha}_{2}, \ldots, \boldsymbol{\alpha}_{\kappa}$ are linearly independent over $\mathbb{Q}$.

Lemma 8 says that the successive minima grow in groups of $\kappa$. This suggests that they are coming from lower-dimensional subspaces. We will follow through with this line of thought.

Let $S$ be an $l$-dimensional subspace of $K^{n}$ and let $\mathfrak{B}$ be a fractional ideal. Let $\lambda_{1} \leq \lambda_{2} \leq \cdots \leq \lambda_{l \kappa}$ be the successive minima of $\rho(\mathfrak{B} I(S))$. We define $i$-dimensional subspaces $S_{i} \subset S$ and minima $\mu_{1} \leq \mu_{2} \leq \cdots \leq \mu_{l}$, as follows: $S_{0}=\{\mathbf{0}\}$, and recursively

$$
\begin{aligned}
\mu_{i+1}=\min \left\{\lambda_{j}:\right. & \text { there exists an } \boldsymbol{\alpha}_{i+1} \in \mathfrak{B} I(S) \\
& \text { with } \left.\boldsymbol{\alpha}_{i+1} \notin S_{i} \text { and }\left\|\rho\left(\boldsymbol{\alpha}_{i+1}\right)\right\|=\lambda_{j}\right\}
\end{aligned}
$$

and

$$
S_{i+1}=S_{i} \oplus K \boldsymbol{\alpha}_{i+1} \quad \text { for } 0 \leq i \leq l-1 .
$$

These subspaces are not necessarily uniquely defined by these conditions; at each stage one may need to make a choice.

Lemma 9. Let $S, \mathfrak{B}$, and $\mu_{1}, \mu_{2}, \ldots, \mu_{l}$ be as above. Let $1 \leq i \leq l$ and let $\lambda_{1}^{\prime} \leq \lambda_{2}^{\prime} \leq \cdots \leq \lambda_{i \kappa}^{\prime}$ be the successive minima of $\rho\left(\mathfrak{B I}\left(S_{i}\right)\right)$. Then

$$
\lambda_{(i-1) \kappa+j}^{\prime} \ll \mu_{i} \leq \lambda_{(i-1) \kappa+j}^{\prime} \quad \text { for } 1 \leq j \leq \kappa .
$$

Proof. Let $\alpha_{1}, \alpha_{2}, \ldots, \alpha_{l}$ be as above in the definition of the $\mu_{i}$ 's. Let $\beta_{1}, \beta_{2}, \ldots, \beta_{\kappa}$ be a $\mathbb{Z}$-basis for $\mathfrak{O}_{K}$. Then, for $1 \leq j \leq \kappa$ and $1 \leq k \leq i$,

$$
\beta_{j} \boldsymbol{\alpha}_{k} \in S_{i} \text { and }\left\|\rho\left(\beta_{j} \boldsymbol{\alpha}_{k}\right)\right\| \ll\left\|\rho\left(\boldsymbol{\alpha}_{i}\right)\right\|=\mu_{i},
$$

by Lemma 8 and the construction of $S_{i}$. This shows that

$$
\lambda_{(i-1) \kappa+j}^{\prime} \leq \lambda_{i \kappa}^{\prime} \ll \mu_{i} \quad \text { for } 1 \leq j \leq \kappa .
$$

Next, for $1 \leq k \leq(i-1) \kappa+1$, pick $\mathbf{Y}_{k} \in \rho\left(\mathfrak{B} I\left(S_{i}\right)\right)$, linearly independent over $\mathbb{Q}$, satisfying $\left\|\mathbf{Y}_{k}\right\|=\lambda_{k}^{\prime}$. Say $\mathbf{Y}_{k}=\rho\left(\boldsymbol{\beta}_{k}\right)$. Since $\mathbf{Y}_{1}, \mathbf{Y}_{2}, \ldots, \mathbf{Y}_{(i-1) \kappa+1}$ span a subspace of dimension $(i-1) \kappa+1>(i-1) \kappa$ in $\mathbb{E}^{n \kappa}$, there must be $\boldsymbol{\beta}_{1}^{\prime}, \boldsymbol{\beta}_{2}^{\prime}, \ldots, \boldsymbol{\beta}_{i}^{\prime}$ among the $\boldsymbol{\beta}_{k}$ 's which are linearly independent over $K$. Thus, there are $i$ linearly independent points $\boldsymbol{\beta}_{1}^{\prime}, \boldsymbol{\beta}_{2}^{\prime}, \ldots, \boldsymbol{\beta}_{i}^{\prime} \in \mathfrak{B} I\left(S_{i}\right)$, satisfying $\left\|\rho\left(\boldsymbol{\beta}_{k}^{\prime}\right)\right\| \leq \lambda_{(i-1) \kappa+1}^{\prime}$ for $1 \leq k \leq i$. This implies $\mu_{i} \leq \lambda_{(i-1) \kappa+j}^{\prime}$ for $1 \leq j \leq \kappa$.

We now have a workable method for dealing with the lattices occurring in Lemma 7.

Lemma 10. Let $S$ be an $(n-d)$-dimensional subspace of $K^{n-1}$. Let $\mathfrak{B}$ be any fractional ideal. For $1 \leq j \leq \kappa$ and $1 \leq i \leq n-d-1$,

$$
N(\mathfrak{B})^{i+j / \kappa} H\left(S_{i}\right)\left(\frac{H\left(S_{i+1}\right)}{H\left(S_{i}\right)}\right)^{j / \kappa} \gg \ll \operatorname{det}\left(\rho(\mathfrak{B} I(S))^{-[(n-d-i) \kappa-j]}\right) .
$$


Proof. Let $\lambda_{1}^{\prime} \leq \lambda_{2}^{\prime} \leq \cdots \leq \lambda_{i \kappa}^{\prime}$ be the successive minima of $\rho\left(\mathfrak{B I}\left(S_{i}\right)\right)$, let $\lambda_{1}^{\prime \prime} \leq \lambda_{2}^{\prime \prime} \leq \cdots \leq \lambda_{(i+1) \kappa}^{\prime \prime}$ be the successive minima of $\rho\left(\mathfrak{B} I\left(S_{i+1}\right)\right)$, and let $\lambda_{1} \leq \lambda_{2} \leq \cdots \leq \lambda_{(n-d) \kappa}$ be the successive minima of $\rho(\mathfrak{B} I(S))$. By Lemma 9 , with $l=n-d$ and $n-1$ in place of $n$,

$$
\begin{aligned}
\prod_{k=1}^{i \kappa} \lambda_{k}^{\prime}\left(\frac{\prod_{k=1}^{(i+1) \kappa} \lambda_{k}^{\prime \prime}}{\prod_{k=1}^{i \kappa} \lambda_{k}^{\prime}}\right)^{j / \kappa} & \gg \ll \prod_{k=1}^{i \kappa} \lambda_{k}^{\prime}\left(\prod_{k=i \kappa+1}^{(i+1) \kappa} \lambda_{k}^{\prime \prime}\right)^{j / \kappa} \gg \ll\left(\prod_{k=1}^{i \kappa} \lambda_{k}^{\prime}\right)\left(\lambda_{i \kappa+1}^{\prime \prime}\right)^{j} \\
& \gg\left(\prod_{k=1}^{i \kappa} \lambda_{k}\right)\left(\lambda_{i \kappa+1}\right)^{j} \gg \ll \prod_{k=1}^{i \kappa+j} \lambda_{k} .
\end{aligned}
$$

But by Theorem 2, Lemma 1, and Minkowski's theorem,

$$
\begin{aligned}
\prod_{k=1}^{i \kappa} \lambda_{k}^{\prime}\left(\frac{\prod_{k=1}^{(i+1) \kappa} \lambda_{k}^{\prime \prime}}{\prod_{k=1}^{i \kappa} \lambda_{k}^{\prime}}\right)^{j / \kappa} & \gg \ll N(\mathfrak{B})^{i} H\left(S_{i}\right)\left(\frac{N(\mathfrak{B})^{i+1} H\left(S_{i+1}\right)}{N(\mathfrak{B})^{i} H\left(S_{i}\right)}\right)^{j / \kappa} \\
& =N(\mathfrak{B})^{i+j / \kappa} H\left(S_{i}\right)\left(\frac{H\left(S_{i+1}\right)}{H\left(S_{i}\right)}\right)^{j / \kappa}
\end{aligned}
$$

and

$$
\prod_{k=1}^{i \kappa+j} \lambda_{k} \gg \ll \operatorname{det}\left(\rho(\mathfrak{B} I(S))^{-[(n-d-i) \kappa-j]}\right) .
$$

Lemma 10 is fairly sharp. However, we will not need to use quite so strong a result to prove Theorem 1. We now state our final version of the Main Error Term, the version we will compute below.

Lemma 11. The Main Error Term is « the maximum over $1 \leq i \leq n-d$ and $1 \leq j \leq \kappa$ of

$\sum_{\mathfrak{A}} \sum_{\mathfrak{C} \mid \mathfrak{A}} \sum_{T} \sum_{S} V(\mathfrak{A}, H(S), B,[n-d-(i-1)] \kappa-j) N\left(\mathfrak{A} \mathfrak{C}^{-1}\right)^{n-d-i+1-j / \kappa} \frac{H(S)}{H(T)^{j / \kappa}}$,

where the first sum is over integral ideals $\mathfrak{A}$ satisfying $N(\mathfrak{A}) \leq B$, the third sum is over $i$-dimensional subspaces $T \subset K^{n-1}$ satisfying $H(T) \leq(B / N(\mathfrak{A}))^{i /(n-d)}$, and the last sum is over $(n-d)$-dimensional subspaces $S \subset K^{n-1}$ satisfying $H(S) \leq B / N(\mathfrak{A})$ and $T=S_{i}$, where $S_{i}$ is defined with respect to the ideal $\mathfrak{A C} \mathfrak{C}^{-1}$

Proof. Lemma 10 implies that, for $S$ an $(n-d)$-dimensional subspace of $K^{n-1}$,

$$
\left[\operatorname{det}\left(\rho\left(\mathfrak{A C} \mathfrak{C}^{-1} I(S)\right)^{-[n-d-(i-1)] \kappa+j}\right)\right]^{-1} \ll N\left(\mathfrak{A C} \mathfrak{C}^{-1}\right)^{-(i-1)-j / \kappa} H\left(S_{i}\right)^{-j / \kappa}
$$

for $1 \leq i \leq n-d-1$ and $1 \leq j \leq \kappa$. Hence, by Lemma 7, the Main Error Term is

$$
\begin{gathered}
\ll \sum_{\mathfrak{A}} \sum_{\mathfrak{E} \mid \mathfrak{A}} \sum_{S} \sum_{i=1}^{n-d} \sum_{j=1}^{\kappa} V(\mathfrak{A}, H(S), B,[n-d-(i-1)] \kappa-j) \\
\cdot N\left(\mathfrak{A C}^{-1}\right)^{n-d-i+1-j / \kappa} \frac{H(S)}{H\left(S_{i}\right)^{j / \kappa}} .
\end{gathered}
$$


By Lemma 9 , if $\lambda_{1}^{\prime} \leq \lambda_{2}^{\prime} \leq \cdots \leq \lambda_{i \kappa}^{\prime}$ denote the successive minima of

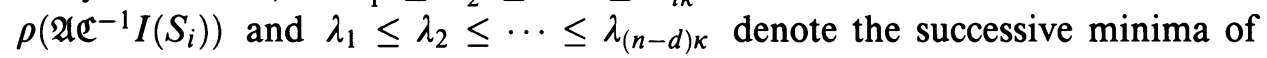
$\rho\left(\mathfrak{A C}^{-1} I(S)\right)$, then

$$
\prod_{k=1}^{i \kappa} \lambda_{k}^{\prime} \ll\left(\prod_{k=1}^{(n-d) \kappa} \lambda_{k}\right)^{i /(n-d)}
$$

Hence, by Minkowski's theorem, Theorem 2, and Lemma 1,

$$
N\left(\mathfrak{A C} \mathfrak{C}^{-1}\right)^{i} H\left(S_{i}\right) \ll\left(N\left(\mathfrak{A C} \mathfrak{C}^{-1}\right)^{n-d} H(S)\right)^{i /(n-d)}
$$

and $H\left(S_{i}\right) \ll H(S)^{i /(n-d)}$. The lemma follows.

\section{SOME TECHNICAL LEMMAS}

In estimating the Main Error Term and the Main Term, we will often make estimates by partial summation. All of these estimates follow the same general pattern, so we carry out the arguments here and simply quote the following result when needed.

Lemma 12. Let $\mathfrak{S}$ be a set together with a function $g$ taking $\mathfrak{S}$ to the ray $[1, \infty)$. Suppose the cardinality of the inverse image of $[1, x]$ satisfies

$$
\operatorname{card}\left\{g^{-1}([1, x])\right\}=c_{1} x^{c_{2}}+O\left(x^{c_{3}}\right),
$$

where $c_{1}, c_{2}$, and $c_{3}$ are positive constants. Let $M \geq 1$ and let $F$ be a $C^{1}$-function on $(0, M)$ such that $F^{\prime}(x) \leq 0$ and increasing on $(0, M)$. Then

$$
\sum_{w \in \mathfrak{S}} F(g(w)) \ll \int_{0}^{M} x^{c_{2}-1} F(x) d x,
$$

and if $F(M)=0$, then

$$
\sum_{w \in \mathfrak{S}} F(g(w))=-c_{1} \int_{0}^{M} x^{c_{2}} F^{\prime}(x) d x+O\left(\int_{0}^{2} x^{c_{2}-1} F(x) d x-\int_{2}^{M} x^{c_{3}} F^{\prime}(x) d x\right),
$$

where the sums are over elements $w \in \mathfrak{S}$ with $g(w) \leq M$ and the constant implicit in the $O$ and $\ll$ notation depends on $c_{1}$ and $c_{2}$.

Proof. Let $M=s+t$, where $s \in \mathbb{Z}^{+}$and $t \in[0,1)$. Define $P_{1}(x)$ to be the number of $w \in \mathfrak{S}$ with $x<g(w) \leq x+1$ and $P_{2}(x)=P_{1}(x-1)$. Suppose $F(M)=0$. Since $g(w) \geq 1$ and $F$ is decreasing,

$$
\begin{aligned}
&(-1)^{j} \sum_{w \in \mathfrak{S}} F(g(w)) \geq(-1)^{j}( P_{j}(t) F(1)+P_{j}(t+1) F(t+1) \\
&\left.+\cdots+P_{j}(s+t-1) F(s+t-1)\right) \\
&=(-1)^{j}( P_{j}(t)(F(1)-F(t+1)) \\
&+\left(P_{j}(t)+P_{j}(t+1)\right)(F(t+1)-F(t+2)) \\
&+\cdots+\left(P_{j}(t)+P_{j}(t+1)+\cdots+P_{j}(s+t-1)\right) \\
&\cdot(F(s+t-1)-F(s+t))),
\end{aligned}
$$

for $j$ equaling 1 or 2 , since $F(s+t)=0$. 
Define $R_{1}(x)$ to be the number of $w \in \mathfrak{S}$ with $g(w) \leq x+1$, and define $R_{2}(x)=R_{1}(x-2)$. Since $F^{\prime}(x)$ is increasing, the mean value theorem gives $\sum_{w \in \mathfrak{S}} F(g(w)) \leq-R_{1}(t) F^{\prime}(1)-R_{1}(t+1) F^{\prime}(t+1)-\cdots-R_{1}(s+t-1) F^{\prime}(s+t-1)$ and

$$
\begin{gathered}
\sum_{w \in \mathfrak{S}} F(g(w)) \geq- \\
-R_{2}(t+2) F^{\prime}(t+2)-R_{2}(t+3) F^{\prime}(t+3) \\
-\cdots-R_{2}(s+t-1) F^{\prime}(s+t-1) .
\end{gathered}
$$

This gives

$$
\begin{aligned}
& \left|\sum_{w \in \mathfrak{S}} F(g(w))+c_{1} \sum_{j=2}^{s-1}(t+j)^{c_{2}} F^{\prime}(t+j)\right| \\
& \quad \ll R_{1}(t) F^{\prime}(1)-R_{1}(t+1) F^{\prime}(t+1)-\sum_{j=2}^{s-1}(t+j)^{c_{3}} F^{\prime}(t+j) .
\end{aligned}
$$

Since

$$
\begin{aligned}
& -R_{1}(t) F^{\prime}(1)-R_{1}(t+1) F^{\prime}(t+1) \ll-\int_{0}^{2} x^{c_{2}} F^{\prime}(x) d x \\
& =\left.x^{c_{2}} F(x)\right|_{0} ^{2}+c_{2} \int_{0}^{2} x^{c_{2}-1} F(x) d x \ll \int_{0}^{2} x^{c_{2}-1} F(x) d x,
\end{aligned}
$$

the second part of the lemma follows. For the first part, we have

$$
\begin{aligned}
\sum_{w \in \mathfrak{S}} F(g(w)) \leq & -R_{1}(t) F^{\prime}(1)-R_{1}(t+1) F^{\prime}(t+1) \\
& -\cdots-R_{1}(s+t-1) F^{\prime}(s+t-1)+R_{1}(s+t) F(s+t) \\
\ll & -\int_{1 / 2}^{M} x^{c_{2}} F^{\prime}(x) d x+M^{c_{2}} F(M) \\
= & -\left.x^{c_{2}} F(x)\right|_{1 / 2} ^{M}+\int_{1 / 2}^{M} x^{c_{2}-1} F(x) d x+M^{c_{2}} F(M) \\
\ll & \int_{0}^{M} x^{c_{2}-1} F(x) d x .
\end{aligned}
$$

For $m$ a positive rational integer, define

$$
\begin{aligned}
\Sigma_{m}=\left\{\left(n_{1}, c_{1}, n_{2}, c_{2}, \ldots, n_{m}, c_{m}\right):\right. & c_{i} \in\{1,2\} \\
& \text { and } \left.c_{i} \leq n_{i} \leq c_{i}(n-d) \text { for all } i\right\} .
\end{aligned}
$$

For $\sigma \in \Sigma_{m}$ and $x \in(0,1]$, define

$$
f_{\sigma}(x)=x \int \cdots \int_{D_{\sigma}(x)} \prod_{j=1}^{m} u_{j}^{n_{j}-1} d u_{j},
$$

where $D_{\sigma}(x)$ is given by $\prod_{j=1}^{m}\left(u_{j}^{2}+1\right)^{c_{j} / 2} \leq 1 / x$ and $u_{j} \geq 0$ for all $j$. Such integrals will appear in the summands of

$$
V(\mathfrak{A}, H(S), B,[n-d-(i-1)] \kappa-j) .
$$


Lemma 13. Let $\sigma \in \Sigma_{m}$. Unless $\sigma=(1,1)$, we have $f_{\sigma}^{\prime}(x) \leq 0$ and increasing on $(0,1]$.

Proof. We will prove the lemma by induction on $m$. If $m=1$, then

$$
\begin{aligned}
f_{\sigma}(x) & =x \int_{0}^{\left(x^{-2 / c_{1}}-1\right)^{1 / 2}} u_{1}^{n_{1}-1} d u_{1}=\frac{x}{n_{1}}\left(x^{-2 / c_{1}}-1\right)^{n_{1} / 2} \\
f_{\sigma}^{\prime}(x) & =\frac{1}{n_{1}}\left(x^{-2 / c_{1}}-1\right)^{n_{1} / 2-1}-\frac{1}{c_{1}} x^{-2 / c_{1}}\left(x^{-2 / c_{1}}-1\right)^{n_{1} / 2-1} \\
& =\frac{1}{n_{1}}\left(x^{-2 / c_{1}}-1\right)^{n_{1} / 2-1}\left(x^{-2 / c_{1}}\left(1-\frac{n_{1}}{c_{1}}\right)-1\right)
\end{aligned}
$$

and

$$
\begin{aligned}
f_{\sigma}^{\prime \prime}(x)= & \frac{n_{1}-2}{c_{1} n_{1}} x^{2 / c_{1}-1}\left(x^{-2 / c_{1}}-1\right)^{n_{1} / 2-1}\left(1-x^{-2 / c_{1}}\left(1-\frac{n_{1}}{c_{1}}\right)\right) \\
& +\frac{2}{c_{1}}\left(\frac{n_{1}}{c_{1}}-1\right) x^{-2 / c_{1}-1}\left(x^{-2 / c_{1}}-1\right)^{n_{1} / 2-1},
\end{aligned}
$$

proving the lemma in this case, since $n_{1} \geq 2$.

Now suppose $m>1$ and that the lemma holds for $m-1$. Let $\sigma^{*}=$ $\left(n_{2}, c_{2}, \ldots, n_{m}, c_{m}\right)$. Unless $\sigma=(1,1,1,1)$, the lemma holds, without loss of generality, for $f_{\sigma^{*}}$. Suppose $\sigma \neq(1,1,1,1)$. We have

$$
\begin{aligned}
f_{\sigma}(x) & =\int_{0}^{\left(x^{-2 / c_{1}}-1\right)^{1 / 2}} u_{1}^{n_{1}-1}\left(u_{1}^{2}+1\right)^{-c_{1} / 2} f_{\sigma^{*}}\left(x\left(u_{1}^{2}+1\right)^{c_{1} / 2}\right) d u_{1} \\
& =\frac{1}{c_{1}} \int_{x}^{1}\left(v^{-2 / c_{1}}-1\right)^{n_{1} / 2-1} v^{-2 / c_{1}} f_{\sigma^{*}}\left(\frac{x}{v}\right) d v,
\end{aligned}
$$

where $v=\left(u_{1}^{2}+1\right)^{-c_{1} / 2}$. Since $f_{\sigma}^{\prime \prime}$ certainly exists, we have $f_{\sigma}^{\prime}(x)$ is

$$
\begin{aligned}
& \frac{1}{c_{1}} \lim _{h \rightarrow 0^{-}} \frac{1}{h}\left[\int_{x+h}^{1}\left(v^{-2 / c_{1}}-1\right)^{n_{1} / 2-1} v^{-2 / c_{1}} f_{\sigma^{*}}\left(\frac{x+h}{v}\right) d v\right. \\
& \quad-\int_{x}^{1}\left(v^{-2 / c_{1}}-1\right)^{n_{1} / 2-1} v^{-2 / c_{1}} \\
& \left.\quad \times\left(f_{\sigma^{*}}\left(\frac{x+h}{v}\right)-f_{\sigma^{*}}\left(\frac{x+h}{v}\right)+f_{\sigma^{*}}\left(\frac{x}{v}\right)\right) d v\right] \\
& =\frac{1}{c_{1}}\left[\lim _{h \rightarrow 0^{-}} \frac{1}{h} \int_{x+h}^{x}\left(v^{-2 / c_{1}}-1\right)^{n_{1} / 2-1} v^{-2 / c_{1}} f_{\sigma^{*}}\left(\frac{x+h}{v}\right) d v\right. \\
& \left.\quad+\lim _{h \rightarrow 0^{-}} \frac{1}{h} \int_{x}^{1}\left(v^{-2 / c_{1}}-1\right)^{n_{1} / 2-1} v^{-2 / c_{1}}\left(f_{\sigma^{*}}\left(\frac{x+h}{v}\right)-f_{\sigma^{*}}\left(\frac{x}{v}\right)\right) d v\right] \\
& =\frac{1}{c_{1}}\left[-\left(x^{-2 / c_{1}}-1\right)^{n_{1} / 2-1} x^{-2 / c_{1}} f_{\sigma^{*}}(1)\right. \\
& \left.\quad+\int_{x}^{1}\left(v^{-2 / c_{1}}-1\right)^{n_{1} / 2-1} v^{-2 / c_{1}-1} f_{\sigma^{*}}^{\prime}\left(\frac{x}{v}\right) d v\right]
\end{aligned}
$$


since $f_{\sigma^{*}}^{\prime}(x / v) \leq 0$ on $[x, 1]$. By the same type of argument, we have $f_{\sigma}^{\prime \prime}(x)$ is equal to

$$
\begin{aligned}
& \frac{1}{c_{1}}\left[-\left(x^{-2 / c_{1}}-1\right)^{n_{1} / 2-1} x^{-2 / c_{1}-1} f_{\sigma^{*}}^{\prime}(1)\right. \\
& \left.\quad+\int_{x}^{1}\left(v^{-2 / c_{1}}-1\right)^{n_{1} / 2-1} v^{-2 / c_{1}-2} f_{\sigma^{*}}^{\prime \prime}\left(\frac{x}{v}\right) d v\right] \geq 0 .
\end{aligned}
$$

It remains to prove the lemma when $\sigma=(1,1,1,1)$. In this case, an easy computation gives

$$
f_{\sigma}(x)=\int_{0}^{\left(x^{-2}-1\right)^{1 / 2}}\left(\left(u^{2}+1\right)^{-1}-x^{2}\right)^{1 / 2} d u .
$$

Arguing as above, we get

$$
\begin{aligned}
f_{\sigma}^{\prime}(x) & =-x \int_{0}^{\left(x^{-2}-1\right)^{1 / 2}}\left(\left(u^{2}+1\right)^{-1}-x^{2}\right)^{-1 / 2} d u \\
& =-\int_{0}^{\left(x^{-2}-1\right)^{1 / 2}}\left(\left(u^{2}+1\right)^{-1} x^{-2}-1\right)^{-1 / 2} d u,
\end{aligned}
$$

which shows the lemma in this case.

Lemma 14. For $u>v-1>0$

$$
\int_{0}^{\infty} \frac{x^{u-v-1}}{\left(x^{2}+1\right)^{u / 2}} d x=\frac{u V(u)}{(u-v) V(u-v) v V(v)}
$$

Proof. Let $y=\left(x^{2}+1\right)^{-1 / 2}$, so that $\left(y^{-2}-1\right)^{1 / 2}=x$ and $x d x=-y^{-3} d y$. The integral is then

$$
-\int_{1}^{0}\left(y^{-2}-1\right)^{(u-v-2) / 2} y^{u} y^{-3} d y=\int_{0}^{1}\left(1-y^{2}\right)^{(u-v) / 2-1} y^{v-1} d y
$$

Now let $z=y^{2}$. Then

by the theory of gamma functions.

$$
\begin{aligned}
\int_{0}^{1}\left(1-y^{2}\right)^{(u-v) / 2-1} y^{v-1} d y & =\frac{1}{2} \int_{0}^{1}(1-z)^{(u-v) / 2-1} z^{v / 2-1} d z \\
& =\frac{1}{2} \frac{\Gamma\left(\frac{u-v}{2}\right) \Gamma\left(\frac{v}{2}\right)}{\Gamma\left(\frac{u}{2}\right)},
\end{aligned}
$$

Using $V(m)=2 \pi^{m / 2} / m \Gamma(m / 2)$ gives the result.

\section{THE CASE $n=2$ OF THEOREM 1}

We will assume in this section that $\kappa \neq 1$, i.e., that $K$ is not $\mathbb{Q}$. For this case, we may simply quote [12, Theorem 1].

In the case $n=2$ of Theorem 1 , we have $d=1$. Then there is only one $(d-1)$-dimensional subspace of $K^{n-1}$, namely $\{0\}$. Since $H(\{0\})=1$, we get the Main Term is

$$
\begin{array}{r}
\sum_{\mathfrak{A}} \sum_{\mathfrak{C} \mid \mathfrak{A}} \mu(\mathfrak{C}) \frac{V(\mathfrak{A}, 1, B, \kappa)}{\operatorname{det}\left(T^{\prime}\left(\mathfrak{C} \mathfrak{A}^{-1}\right)\right)} \quad(\text { letting } \mathfrak{A}=\mathfrak{B C}) \\
\quad=\sum_{\mathfrak{C}} \mu(\mathfrak{C}) \sum_{\mathfrak{B}} V(\mathfrak{B C}, 1, B, \kappa) N(\mathfrak{B}) \frac{2^{r_{2}}}{\sqrt{|\delta|}},
\end{array}
$$


where the first sum is over integral ideals $\mathfrak{C}$ with norm $\leq B$ and the second sum is over integral ideals $\mathfrak{B}$ with norm $\leq B / N(\mathfrak{C})$, since

$$
\operatorname{det}\left(T^{\prime}(\mathfrak{B})\right)=\operatorname{det}(T \circ \rho(\mathfrak{B}))=2^{r_{2}} \operatorname{det}(\rho(\mathfrak{B}))=\frac{2^{r_{2}}}{\sqrt{|\delta|}} N(\mathfrak{B})
$$

by Lemma 1 and Theorem 2. Now $V(\mathfrak{B C}, 1, B, \kappa)$ is the volume of the domain

$$
\left\{\mathbf{Y} \in \mathbb{R}^{\kappa}: \prod_{i=1}^{r_{1}+r_{2}}\left(\left\|\mathbf{y}_{i}\right\|^{2}+1\right)^{e_{i} / 2} \leq \frac{B}{N(\mathfrak{B}) N(\mathfrak{C})}\right\}
$$

Letting $u_{i}=\left\|\mathbf{y}_{i}\right\|$, we have

(11) $\sum_{\mathfrak{B}} V(\mathfrak{B C}, 1, B, \kappa) N(\mathfrak{B})=\frac{B}{N(\mathfrak{C})} V(1)^{r_{1}}(2 V(2))^{r_{2}} \sum_{\mathfrak{B}} f_{\sigma}(N(\mathfrak{B}) N(\mathfrak{C}) / B)$,

where $\sigma=\left(e_{1}, 1, \ldots, e_{r_{1}}, 1, e_{r_{1}+1}, 2, \ldots, e_{r_{1}+r_{2}}, 2\right)$ (see [3, Chapter 2]). Define $F(x)=f_{\sigma}(x N(\mathfrak{C}) / B)$. By Lemma 13, since $\kappa \neq 1, \quad F$ satisfies the hypotheses of Lemma 12. By the Dedekind-Weber theorem (see [6]), the number of integral ideals with norm $\leq M$ is $h \chi M+O\left(M^{1-1 / \kappa}\right)$, where

$$
\chi=\frac{2^{r_{1}+r_{2}} \pi^{r_{2}} R}{w \sqrt{|\delta|}}
$$

By Lemma 12 , letting $M=B / N(\mathfrak{C})$, we have

$$
\sum_{\mathfrak{B}} F(N(\mathfrak{B}))=-h \chi \int_{0}^{M} x F^{\prime}(x) d x+O\left(\int_{0}^{2} F(x) d x-\int_{2}^{M} x^{1-1 / \kappa} F^{\prime}(x) d x\right) .
$$

Integration by parts yields

$$
\begin{aligned}
-h \chi \int_{0}^{M} x F^{\prime}(x) d x & =-\left.h \chi x F(x)\right|_{0} ^{M}+h \chi \int_{0}^{M} F(x) d x \\
& =h \chi \int_{0}^{M} x \int \cdots \int_{D_{\sigma}(x / M)} \prod_{j=1}^{r_{1}+r_{2}} d u_{j} d x \\
& =h \chi M \int_{0}^{1} y \int \cdots \int_{D_{\sigma}(y)} \prod_{j=1}^{r_{1}+r_{2}} d u_{j} d y \\
& =h \chi M \int_{0}^{\infty} \cdots \int_{0}^{\infty} \prod_{j=1}^{r_{1}+r_{2}} \int_{0}^{\prod_{j=1}^{r_{1}+r_{2}}\left(u_{j}^{2}+1\right)^{-e_{j} / 2}} y d y \\
& =\frac{h}{2} \chi M \prod_{j=1}^{r_{1}+r_{2}} \int_{0}^{\infty} u_{j}^{e_{j}-1}\left(u_{j}^{2}+1\right)^{-e_{j}} d u_{j} \\
& =\frac{h}{2} \chi \frac{B}{N(\mathfrak{C})}\left(\frac{2 V(2)}{V(1) V(1)}\right)^{r_{1}}\left(\frac{V(4)}{V(2) V(2)}\right)^{r_{2}},
\end{aligned}
$$

by Lemma 14. Similar computations give the first integral in the error term in $(12)$ is $\ll 1$ and the second is $\ll(B / N(\mathfrak{C}))^{1-1 / \kappa}$. We thus have, by (11) 
and (12),

$$
\begin{aligned}
\sum_{\mathfrak{C}} \mu(\mathfrak{C}) & \sum_{\mathfrak{B}} V(\mathfrak{B C}, 1, B, \kappa) N(\mathfrak{B}) \frac{2^{r_{2}}}{\sqrt{|\delta|}} \\
= & \frac{h R}{w}\left(\frac{2^{r_{2}}}{\sqrt{|\delta|}}\right)^{2} \frac{1}{2} 2^{r_{1}+r_{2}}(V(2))^{r_{1}}(V(4))^{r_{2}} B^{2} \sum_{\mathfrak{C}} \mu(\mathfrak{C}) N(\mathfrak{C})^{-2} \\
& +O\left(B^{2-1 / \kappa} \sum_{\mathfrak{C}} N(\mathfrak{C})^{-(2-1 / \kappa)}\right)
\end{aligned}
$$

Now

$$
\begin{aligned}
\sum_{N(\mathfrak{C}) \leq B} \mu(\mathfrak{C}) N(\mathfrak{C})^{-2} & =\sum_{\mathfrak{C} \subset \mathfrak{O}_{K}} \mu(\mathfrak{C}) N(\mathfrak{C})^{-2}-\sum_{N(\mathfrak{C})>B} \mu(\mathfrak{C}) N(\mathfrak{C})^{-2} \\
& =\frac{1}{\zeta_{K}(2)}+O\left(B^{-1}\right)
\end{aligned}
$$

and

$$
\sum_{N(\mathfrak{C}) \leq B} N(\mathfrak{C})^{-(2-1 / \kappa)} \leq \zeta_{K}(2-1 / \kappa)=O(1)
$$

Thus, the Main Term is $a(2,1) B^{2}+O\left(B^{n-b(2,1)}\right)$.

Now the Main Error Term is $\ll$ the maximum over $1 \leq j \leq \kappa$ of

$$
\begin{gathered}
\sum_{\mathfrak{A}} \sum_{\mathfrak{C} \mid \mathfrak{A}} V(\mathfrak{A}, 1, B, \kappa-j) N\left(\mathfrak{A} \mathfrak{C}^{-1}\right)^{1-j / \kappa} \quad(\text { letting } \mathfrak{A}=\mathfrak{B C}) \\
=\sum_{\mathfrak{C}} \sum_{\mathfrak{B}} V(\mathfrak{B C}, 1, \boldsymbol{B}, \kappa-j) N(\mathfrak{B})^{1-j / \kappa},
\end{gathered}
$$

where the first sum is over integral ideals $\mathfrak{C}$ with norm $\leq B$ and the second sum is over integral ideals $\mathfrak{B}$ with norm $\leq B / N(\mathfrak{C})$, by Lemma 11 . For $j=\kappa$, we have the inner sum in (13) is $\ll B / N(\mathfrak{C})$. For $j<\kappa$, the inner sum in (13) is of the form

$$
\sum_{\mathfrak{B}} \frac{B}{N(\mathfrak{C})} f_{\sigma}\left(\frac{N(\mathfrak{B}) N(\mathfrak{C})}{B}\right) N(\mathfrak{B})^{-j / \kappa}
$$

Letting

$$
F(x)=f_{\sigma}(x N(\mathfrak{C}) / B) x^{-j / \kappa},
$$

we have that $F$ satisfies the hypothesis of Lemma 12, even in the case $\sigma=$ $(1,1)$. Thus, by the Dedekind-Weber theorem and Lemma 12, we have

$$
\sum_{\mathfrak{B}} F(N(\mathfrak{B})) \ll \int_{0}^{B / N(\mathfrak{C})} F(x) d x=\left(\frac{B}{N(\mathfrak{C})}\right)^{1-j / \kappa} \int_{0}^{1} f_{\sigma}(y) y^{-j / \kappa} \ll\left(\frac{B}{N(\mathfrak{C})}\right)^{1-j / \kappa}
$$

by a straightforward computation. So the inner sum is always $\ll(B / N(\mathfrak{C}))^{1-j / \kappa}$. Hence, the Main Error Term is

$$
\ll B^{2-1 / \kappa}
$$

for $\kappa \geq 2$, and Theorem 1 is proven in the case $n=2$. 


\section{Computation of the Main Error Term}

For the remainder of this paper, we will assume $n>2$ and that Theorem 1 holds for $n-1$. We will also exploit the symmetery:

$$
M(n, d, B)=M(n, n-d, B) .
$$

In this section we will prove

Proposition 1. For $d \geq n / 2$ the Main Error Term is $\ll B^{n-1 / \kappa(n-d)}$.

In order to use Lemma 11, we need the following estimate.

Lemma 15. Let $\mathfrak{B}$ be a fractional ideal and let $S^{d}$ be a d-dimensional subspace of $K^{n}$, where $0 \leq d \leq n-2$. Let $H\left(S^{d}\right)=B_{0}$ and $B \geq B_{0}$. Then the number of $(d+i)$-dimensional subspaces $S \supset S^{d}$, where $1 \leq i \leq n-d-1$, i.e., where $1 \leq d+i \leq n-1$, satisfying $H(S) \leq B$ and $S_{d}=S^{d}$ is $\ll B^{n-d} B_{0}^{d+i-n}$. (The ideal $\mathfrak{B}$ is needed for the definition of $S_{d}$, though the bound is independent of it.)

Proof. We use induction on $i$. For $i=1$ we will simply quote [11, Lemma 10], which is actually stronger than the stated result.

Now let $i>1$. Suppose $S \supset S^{d}$ with $H(S) \leq B$ and $S_{d}=S^{d}$. Consider the subspace $S^{\prime}=S_{d+1}$. We show that

$$
H\left(S^{\prime}\right) \ll B_{0}\left(\frac{B}{B_{0}}\right)^{1 / i}
$$

Let $\lambda_{1}^{\prime} \leq \lambda_{2}^{\prime} \leq \cdots \leq \lambda_{d \kappa}^{\prime}$ be the successive minima of $\rho\left(\mathfrak{B I}\left(S^{d}\right)\right)$, let $\lambda_{1}^{\prime \prime} \leq$ $\lambda_{2}^{\prime \prime} \leq \cdots \leq \lambda_{(d+1) \kappa}^{\prime \prime}$ be the successive minima of $\rho\left(\mathfrak{B I}\left(S^{\prime}\right)\right)$, and let $\lambda_{1} \leq \lambda_{2} \leq$ $\cdots \leq \lambda_{(d+i) \kappa}$ be the successive minima of $\rho(\mathfrak{B} I(S))$. By Lemma 10, Lemma 1 , Theorem 2, and Minkowski's theorem,

$$
\begin{aligned}
N(\mathfrak{B})^{d+1} H\left(S^{\prime}\right) & \gg \prod_{j=1}^{(d+1) \kappa} \lambda_{j}^{\prime \prime} \gg \ll \prod_{j=1}^{d \kappa} \lambda_{j}^{\prime} \prod_{j=d \kappa+1}^{(d+1) \kappa} \lambda_{j}^{\prime \prime} \\
& \gg \ll H\left(S^{d}\right) N(\mathfrak{B})^{d} \prod_{j=d \kappa+1}^{(d+1) \kappa} \lambda_{j}^{\prime \prime},
\end{aligned}
$$

giving

We also have

$$
H\left(S^{\prime}\right) \ll H\left(S^{d}\right) N(\mathfrak{B})^{-1} \prod_{j=d \kappa+1}^{(d+1) \kappa} \lambda_{j}^{\prime \prime} .
$$

$$
\begin{aligned}
N(\mathfrak{B})^{d+i} H(S) & \gg \ll \prod_{j=1}^{(d+i) \kappa} \lambda_{j} \gg \ll \prod_{j=1}^{d \kappa} \lambda_{j}^{\prime} \prod_{j=d \kappa+1}^{(d+1) \kappa} \lambda_{j}^{\prime \prime} \prod_{j=(d+1) \kappa+1}^{(d+i) \kappa} \lambda_{j} \\
& \gg \ll H\left(S^{d}\right) N(\mathfrak{B})^{d}\left(\prod_{j=d \kappa+1}^{(d+1) \kappa} \lambda_{j}^{\prime \prime}\right)^{i}
\end{aligned}
$$

giving

$$
N(\mathfrak{B})\left(\frac{H(S)}{H\left(S^{d}\right)}\right)^{1 / i} \gg \prod_{j=d \kappa+1}^{(d+1) \kappa} \lambda_{j}^{\prime \prime} .
$$


We thus have

$$
\begin{aligned}
H\left(S^{\prime}\right) & \ll H\left(S^{d}\right) N(\mathfrak{B})^{-1} \prod_{j=d \kappa+1}^{(d+1) \kappa} \lambda_{j}^{\prime \prime} \\
& \ll H\left(S^{d}\right)\left(\frac{H(S)}{H\left(S^{d}\right)}\right)^{1 / i} \leq B_{0}\left(\frac{B}{B_{0}}\right)^{1 / i},
\end{aligned}
$$

giving (14).

Now suppose a subspace $S^{\prime}$ of dimension $d+1$ is given. By the induction hypothesis, the number of $(d+i)$-dimensional subspaces $S \supset S^{\prime}$ satisfying $H(S) \leq B$ and $S_{d+1}=S^{\prime}$ is $\ll B^{n-(d+1)} H\left(S^{\prime}\right)^{d+i-n}$. We therefore must estimate the sum $\sum_{S^{\prime}} H\left(S^{\prime}\right)^{d+i-n}$ over $(d+1)$-dimensional subspaces $S^{\prime} \supset S^{d}$ with

$$
H\left(S^{\prime}\right) \leq B_{0}\left(\frac{B}{B_{0}}\right)^{1 / i}
$$

We apply Lemma 12 and the case $i=1$, with $F(x)=x^{d+i-n}$ and $M=$ $B_{0}\left(B / B_{0}\right)^{1 / i}$, to get

$$
\begin{aligned}
\sum_{S^{\prime}} H\left(S^{\prime}\right)^{d+1-n} & \ll B_{0}^{d+1-n} \int_{0}^{M} x^{n-d-1} F(x) d x \\
& \ll B_{0}^{d+1-n} \int_{0}^{M} x^{i-1} d x \ll B_{0}^{d+1-n} M^{i}=B B_{0}^{d+i-n} .
\end{aligned}
$$

The lemma follows.

We are now in a position to estimate the Main Error Term. Note that in Lemma 11 , when $i=n-d$, the third sum is irrelevant. We will handle this case later. For now we will concentrate on the case $i<n-d$.

Lemma 16. Let $d \geq n / 2$ and fix $i$ and $j$ with $1 \leq i<n-d$ and $1 \leq j \leq \kappa$, so that $[n-d-(i-1)] \kappa-j>1$. Let $T$ be an $i$-dimensional subspace of $K^{n-1}$. Then

$$
\sum_{S} V(\mathfrak{A}, H(S), B,[n-d-(i-1)] \kappa-j) H(S) \ll H(T)^{1-d}\left(\frac{B}{N(\mathfrak{A})}\right)^{n-i},
$$

where the sum is over $(n-d)$-dimensional subspaces $S \subset K^{n-1}$ satisfying $S_{i}=T$ and $H(S) \leq B / N(\mathfrak{A})$.

Proof. It suffices to show this for each summand of $V(\mathfrak{A}, H(S), B,[n-d-$ $(i-1)] \kappa-j)$. Such a summand will be the volume of a domain of the form

$$
\left\{\mathbf{Y}=\left(\mathbf{y}_{1}, \mathbf{y}_{2}, \ldots, \mathbf{y}_{m}\right): \prod_{j=1}^{r_{1}+r_{2}}\left(\left\|\mathbf{y}_{j}\right\|^{2}+1\right)^{\boldsymbol{e}_{j} / 2} \leq \frac{B}{N(\mathfrak{A}) H(S)}\right\}
$$

where, unless it is $\mathbf{0}, \mathbf{y}_{j} \in \mathbb{R}^{n_{j}}$ and $1 \leq n_{j} \leq e_{j}(n-d)$ for $j=1,2, \ldots, r_{1}+r_{2}$. After the change of variables $u_{j}=\left\|\mathbf{y}_{j}\right\|$, we see that such a summand will be of the form

$$
C \int \cdots \int \prod_{j=1}^{m} u_{j}^{n_{j}-1} d u_{j}
$$


where the domain of integration is given by

$$
\prod_{j=1}^{m}\left(u_{j}^{2}+1\right)^{c_{j} / 2} \leq \frac{B}{N(\mathfrak{A}) H(S)} \quad \text { and } \quad u_{j} \geq 0
$$

where $c_{j} \in\{1,2\}, 1 \leq n_{j} \leq c_{j}(n-d), \quad C$ is a product of measures of unit spheres, and $m \leq r_{1}+r_{2}$. If $n_{j_{0}}=1$ and $c_{j_{0}}=2$ for some $j_{0}$, the integral is made larger by changing $c_{j_{0}}$ to 1 . Thus, for each summand of $V(\mathfrak{A}, H(S), B,[n-d-(i-1)] \kappa-j)$, we are estimating a sum of the form

$$
\sum_{S} \frac{B}{N(\mathfrak{A})} f_{\sigma}\left(\frac{N(\mathfrak{A}) H(S)}{B}\right)
$$

where $\sigma \in \Sigma_{s}, \sigma \neq(1,1)$ for some $s$ between 1 and $r_{1}+r_{2}$. We apply Lemma 12 and Lemma 15 to the sum

$$
\sum_{S} f_{\sigma}\left(\frac{N(\mathfrak{A}) H(S)}{B}\right)
$$

letting $g(x)=H(T)^{1-d} x^{n-i-1}$ and

$$
F(x)=f_{\sigma}\left(x \frac{N(\mathfrak{A})}{B}\right)
$$

We get that this sum is

$$
\begin{aligned}
& \ll H(T)^{1-d} \int_{0}^{B / N(\mathfrak{A})} x^{n-i-2} f_{\sigma}\left(x \frac{N(\mathfrak{A})}{B}\right) d x \\
& =H(T)^{1-d}\left(\frac{B}{N(\mathfrak{A})}\right)^{n-i-1} \int_{0}^{1} y^{n-i-2} f_{\sigma}(y) d y \\
& =H(T)^{1-d}\left(\frac{B}{N(\mathfrak{A})}\right)^{n-i-1} \int_{0}^{\infty} \cdots \int_{0}^{\infty} \int_{0}^{\prod_{j=1}^{m}\left(u_{j}^{2}+1\right)^{-c_{j} / 2}} y^{n-i-1} \prod_{j=1}^{m} u_{j}^{n_{j}-1} d y d u_{j} \\
& =H(T)^{1-d}\left(\frac{B}{N(\mathfrak{A})}\right)^{n-i-1} \prod_{j=1}^{m} \int_{0}^{\infty} u_{j}^{n_{j}-1}\left(u_{j}^{2}+1\right)^{-(n-i) c_{j} / 2} d u_{j} \\
& \ll H(T)^{1-d}\left(\frac{B}{N(\mathfrak{A})}\right)^{n-i-1}\left(1+\prod_{j=1}^{m} \int_{1}^{\infty} u_{j}^{n_{j}-1}\left(u_{j}^{2}+1\right)^{-(n-i) c_{j} / 2} d u_{j}\right) \\
& \ll H(T)^{1-d}\left(\frac{B}{N(\mathfrak{A})}\right)^{n-i-1},
\end{aligned}
$$

since $(n-i)>d \geq n / 2 \geq(n-d) \geq n_{j} / c_{j}$. The lemma follows.

We now apply Lemma 12 and the induction hypothesis to Theorem 1, with $g(x)=x^{n-1}, \quad F(x)=x^{1-d-j / \kappa}$, and $M=(B / N(\mathfrak{A}))^{i /(n-d)}$ to the sum 
$\sum_{T} H(T)^{1-d-j / \kappa}$. We get

$$
\begin{aligned}
\sum_{T} H(T)^{1-d-j / \kappa} & \ll \int_{0}^{M} g^{\prime}(x) F(x) d x \\
& \ll \int_{0}^{M} x^{n-1-d-j / \kappa} d x \ll M^{n-d-j / \kappa} \\
& =\left(\frac{B}{N(\mathfrak{A})}\right)^{(n-d-j / \kappa) i /(n-d)}=\left(\frac{B}{N(\mathfrak{A})}\right)^{i-i j / \kappa(n-d)} .
\end{aligned}
$$

We now consider the case $i=n-d$. By an argument entirely analogous to that in the last section, we have

$$
\sum_{S} V(\mathfrak{A}, H(S), B, \kappa-j) H(S)^{1-j / \kappa} \ll\left(\frac{B}{N(\mathfrak{A})}\right)^{n-j / \kappa},
$$

where the sum is over $(n-d)$-dimensional subspaces $S \subset K^{n-1}$ with $H(S) \leq$ $B / N(\mathfrak{A})$.

By Lemma 16, (15), and (16), the Main Error Term is

$$
\begin{aligned}
& \ll \max \left\{\sum_{\mathfrak{A}} \sum_{\mathfrak{C} \mid \mathfrak{A}} N\left(\mathfrak{A} \mathfrak{C}^{-1}\right)^{n-d-i+1-j / \kappa}\left(\frac{B}{N(\mathfrak{A})}\right)^{n-i j / \kappa(n-d)}\right\} \\
& =\max \left\{B^{n-i j / \kappa(n-d)} \sum_{\mathfrak{A}} N(\mathfrak{A})^{-d-i+1+i j / \kappa(n-d)-j / \kappa} \sum_{\mathfrak{C} \mid \mathfrak{A}} N(\mathfrak{C})^{d-n+i-1+j / \kappa}\right\} \\
& \leq B^{n-1 / \kappa(n-d)} \sum_{\mathfrak{A}} N(\mathfrak{A})^{-d+1-1 / \kappa} \sum_{\mathfrak{C} \mid \mathfrak{A}} 1 .
\end{aligned}
$$

But the number of integral ideals $\mathfrak{C}$ with $\mathfrak{C} \mid \mathfrak{A}$ is $\leq c_{\varepsilon} N(\mathfrak{A})^{\varepsilon}$, where $c_{\varepsilon}$ is a constant depending only on $K$ and $\varepsilon$. Letting $\varepsilon=1 / 2 \kappa$ gives that the Main Error Term is

$$
\begin{aligned}
& \ll B^{n-1 / \kappa(n-d)} \sum_{\mathfrak{A}} N(\mathfrak{A})^{-d+1-1 / 2 \kappa} \\
& <B^{n-1 / \kappa(n-d)} \zeta_{K}(d-1+1 / 2 \kappa) \ll B^{n-1 / \kappa(n-d)},
\end{aligned}
$$

since $d \geq 2$.

\section{Computation of the Main Term}

As in $\S 6$, we will assume $\kappa>1$. In this section we prove Proposition 2. For $n>2$ and $n>d>1$, the Main Term is

$$
a(n, d) B^{n}+O\left(B^{n-1}\right) .
$$

We first consider the sum over subspaces.

Lemma 17. For $n>2$ and $n>d>1$,

$$
\begin{aligned}
\sum_{S} \frac{V(\mathfrak{A}, H(S), B,(n-d) \kappa)}{\operatorname{det}\left(T^{\prime}\left(\left(\mathfrak{C} \mathfrak{A}^{-1}\right)^{n-1}\right)\right)}= & a(n, d) \frac{\zeta_{K}(n)}{\zeta_{K}(d)} N\left(\mathfrak{A} \mathfrak{C}^{-1}\right)^{n-d}\left(\frac{B}{N(\mathfrak{A})}\right)^{n} \\
& +O\left(N\left(\mathfrak{A} \mathfrak{C}^{-1}\right)^{n-d}\left(\frac{B}{N}(\mathfrak{A})\right)^{n-b(n-1, d-1)}\right),
\end{aligned}
$$


where the sum is over $(d-1)$-dimensional subspaces $S \subset K^{n-1}$ of height less than or equal to $B / N(\mathfrak{A})$.

Proof. By (8), we have

$$
\operatorname{det}\left(\pi \circ \rho\left(\left(\mathfrak{C} \mathfrak{A}^{-1}\right)^{n-1}\right)\right)=\left(N\left(\mathfrak{C} \mathfrak{A}^{-1}\right) \sqrt{|\delta|}\right)^{n-d} H(S)^{-1},
$$

giving

$$
\operatorname{det}\left(T^{\prime}\left(\left(\mathfrak{C} \mathfrak{A}^{-1}\right)^{n-1}\right)\right)=\left(\frac{N\left(\mathfrak{C} \mathfrak{A}^{-1}\right) \sqrt{|\delta|}}{2^{r_{2}}}\right)^{n-d} H(S)^{-1}
$$

By definition, $V(\mathfrak{A}, H(S), B,(n-d) \kappa)$ is the volume of the set

$$
\left\{\mathbf{Y} \in \mathbb{R}^{(n-d) \kappa}: \prod_{i=1}^{r_{1}+r_{2}}\left(\left\|\mathbf{y}_{i}\right\|^{2}+1\right)^{\boldsymbol{e}_{i} / 2} \leq \frac{B}{N(\mathfrak{A}) H(S)}\right\} .
$$

Thus, setting $u_{j}=\left\|\mathbf{y}_{j}\right\|$ for $j=1,2, \ldots, r_{1}+r_{2}$, we have

$$
\begin{aligned}
V(\mathfrak{A}, H(S), B,(n-d) \kappa)= & ((n-d) V(n-d))^{r_{1}}(2(n-d) V(2(n-d)))^{r_{2}} \\
& \cdot \frac{B}{N(\mathfrak{A}) H(S)} f_{\sigma}\left(\frac{H(S) N(\mathfrak{A})}{B}\right),
\end{aligned}
$$

where

$$
\sigma=\left(e_{1}(n-d), e_{1}, e_{2}(n-d), e_{2}, \ldots, e_{r_{1}+r_{2}}(n-d), e_{r_{1}+r_{2}}\right) \neq(1,1)
$$

since $\kappa \geq 2$. We then have

$$
\begin{aligned}
& \sum_{S} \frac{V(\mathfrak{A}, H(S), B,(n-d) p)}{\operatorname{det}\left(T^{\prime}\left(\left(\mathfrak{C} \mathfrak{A}^{-1}\right)^{n-1}\right)\right)}=\left(\frac{2^{r_{2}}}{\sqrt{|\delta|}}\right)^{n-d}((n-d) V(n-d))^{r_{1}} \\
& \cdot(2(n-d) V(2(n-d)))^{r_{2}} N\left(\mathfrak{A} \mathfrak{C}^{-1}\right)^{n-d} \frac{B}{N(\mathfrak{A})} \cdot \sum_{S} f_{\sigma}\left(\frac{H(S) N(\mathfrak{A})}{B}\right) .
\end{aligned}
$$

We will estimate $\sum_{S} f_{\sigma}(H(S) N(\mathfrak{A}) / B)$. Define $f(x)=f_{\sigma}(x N(\mathfrak{A}) / B)$. By Lemmas 12 and 13 and the induction hypothesis to Theorem 1 , we have

$$
\begin{aligned}
\sum_{S} f(H(S))= & -a(n-1, d-1) \int_{0}^{B / N(\mathfrak{A})} x^{n-1} f^{\prime}(x) d x \\
& +O\left(\int_{0}^{2} x^{n-2} f(x) d x-\int_{2}^{B / N(\mathfrak{A})} x^{n-1-b(n-1, d-1)} f^{\prime}(x) d x\right) .
\end{aligned}
$$

A straightforward computation gives the integrals in the error term in (18) are $\ll(B / N(\mathfrak{A}))^{n-1-b(n-1, d-1)}$.

It remains to estimate the main term in (18). We have

$$
\begin{aligned}
-\int_{0}^{B / N(\mathfrak{A})} x^{n-1} f^{\prime}(x) d x & =-\left.x^{n-1} f(x)\right|_{0} ^{B / N(\mathfrak{A})}+(n-1) \int_{0}^{B / N(\mathfrak{A})} x^{n-2} f(x) d x \\
& =(n-1) \int_{0}^{B / N(\mathfrak{A})} x^{n-2} f(x) d x
\end{aligned}
$$


Letting $y=x N(\mathfrak{A}) / B$, we have

$$
\begin{aligned}
& \int_{0}^{B / N(\mathfrak{A})} x^{n-2} f(x) d x=\left(\frac{B}{N(\mathfrak{A})}\right)^{n-1} \int_{0}^{1} y^{n-2} f_{\sigma}(y) d y \\
&=\left(\frac{B}{N(\mathfrak{A})}\right)^{n-1} \int_{0}^{1} y^{n-1} \int \cdots \int_{D_{\sigma}(y)} \prod_{j=1}^{r_{1}+r_{2}} u_{j}^{(n-d) e_{j}-1} d u_{j} d y \\
&=\left(\frac{B}{N(\mathfrak{A})}\right)^{n-1} \ldots \int_{0}^{\infty} u_{j}^{e_{j}(n-d)-1} \ldots \int_{0}^{\prod_{j=1}^{r_{1}+r_{2}}\left(u_{j}^{2}+1\right)^{-e_{j} / 2}} y^{n-1} d y d u_{j} \\
&= \frac{1}{n}\left(\frac{B}{N(\mathfrak{A})}\right)^{n-1} \prod_{j=1}^{r_{1}+r_{2}} \int_{0}^{\infty} u_{j}^{(n-d) e_{j}-1}\left(u_{j}^{2}+1\right)^{-e_{j} n / 2} d u_{j} \\
&= \frac{1}{n}\left(\frac{B}{N(\mathfrak{A})}\right)^{n-1}\left(\int_{0}^{\infty} v^{n-d-1}\left(v^{2}+1\right)^{-n / 2} d v\right)^{r_{1}} \\
& \cdot\left(\int_{0}^{\infty} v^{2(n-d)-1}\left(v_{j}^{2}+1\right)^{-n} d v\right)^{r_{2}} \\
&= \frac{1}{n}\left(\frac{B}{N(\mathfrak{A})}\right)^{n-1}\left(\frac{n V(n)}{(n-d) V(n-d) d V(d)}\right)^{r_{1}} \\
& \cdot\left(\frac{n V(2 n)}{2(n-d) V(2(n-d)) d V(2 d)}\right)^{r_{2}},
\end{aligned}
$$

by Lemma 14 . We thus have

$$
\begin{aligned}
\sum_{S} f(H(S)) & =a(n-1, d-1) \frac{(n-1)}{n}\left(\frac{B}{N(\mathfrak{A})}\right)^{n-1} \\
\cdot & \left(\frac{n V(n)}{(n-d) V(n-d) d V(d)}\right)^{r_{1}}\left(\frac{n V(2 n)}{2(n-d) V(2(n-d)) d V(2 d)}\right)^{r_{2}} \\
+ & O\left(\left(\frac{B}{N(\mathfrak{A})}\right)^{n-1-b(n-1, d-1)}\right.
\end{aligned}
$$

Hence, by (17),

$$
\begin{aligned}
\sum_{S} \frac{V(\mathfrak{A}, H(S), B,(n-d) \kappa)}{\operatorname{det}\left(T^{\prime \prime}\left(\mathfrak{C} \mathfrak{A}^{-1}\right)^{n-1}\right)} \\
=a(n-1, d-1)\left(\frac{2^{r_{2}}}{\sqrt{|\delta|}}\right)^{n-d} \frac{(n-1)}{n}\left(\frac{n}{d}\right)^{r_{1}+r_{2}} \\
\\
\cdot\left(\frac{V(n)}{V(d)}\right)^{r_{1}}\left(\frac{V(2 n)}{V(2 d)}\right)^{r_{2}} N\left(\mathfrak{A} \mathfrak{C}^{-1}\right)^{n-d}\left(\frac{B}{N(\mathfrak{A})}\right)^{n} \\
+O\left(N\left(\mathfrak{A} \mathfrak{C}^{-1}\right)^{n-d}\left(\frac{B}{N(\mathfrak{A})}\right)^{n-b(n-1, d-1)}\right) .
\end{aligned}
$$


Since

$$
\begin{gathered}
a(n, d)=a(n-1, d-1)\left(\frac{2^{r_{2}}}{\sqrt{|\delta|}}\right)^{n-d} \frac{(n-1)}{n}\left(\frac{n}{d}\right)^{r_{1}+r_{2}} \\
\cdot\left(\frac{V(n)}{V(d)}\right)^{r_{1}}\left(\frac{V(2 n)}{V(2 d)}\right)^{r_{2}} \frac{\zeta_{K}(d)}{\zeta_{K}(n)}
\end{gathered}
$$

we are done.

Proof of Proposition 2. We first show

$$
\sum_{N(\mathfrak{A}) \leq B} N(\mathfrak{A})^{-d} \sum_{\mathfrak{C} \mid \mathfrak{A}} \mu(\mathfrak{C}) N(\mathfrak{C})^{d-n}=\frac{\zeta_{K}(d)}{\zeta_{K}(n)}+O\left(B^{-1}\right) .
$$

Letting $\mathfrak{A}=\mathfrak{B C}$, we get

$$
\sum_{N(\mathfrak{A}) \leq B} N(\mathfrak{A})^{-d} \sum_{\mathfrak{C} \mid \mathfrak{A}} \mu(\mathfrak{C}) N(\mathfrak{C})^{n-d}=\sum_{N(\mathfrak{C}) \leq B} \mu(\mathfrak{C}) N(\mathfrak{C})^{-n} \sum_{N(\mathfrak{B}) \leq B / N(\mathfrak{C})} N(\mathfrak{B})^{-d} .
$$

Using the number of integral ideals of norm $\leq M$ is $O(M)$ and partial summation estimates, we get

$$
\begin{gathered}
\sum_{N(\mathfrak{B}) \leq B / N(\mathfrak{C})} N(\mathfrak{B})^{-d}=\zeta_{K}(d)-\sum_{N(\mathfrak{B})>B / N(\mathfrak{C})} N(\mathfrak{B})^{-d} \\
=\zeta_{K}(d)+O\left((B / N(\mathfrak{C}))^{1-d}\right), \\
\sum_{N(\mathfrak{C}) \leq B} \mu(\mathfrak{C}) N(\mathfrak{C})^{-n}=\sum_{\mathfrak{C} \subset \mathfrak{D}_{K}} \mu(\mathfrak{C}) N(\mathfrak{C})^{-n}-\sum_{N(\mathfrak{C})>B} \mu(\mathfrak{C}) N(\mathfrak{C})^{-n} \\
=\frac{1}{\zeta_{K}(n)}+O\left(B^{1-n}\right),
\end{gathered}
$$

and

$$
\sum_{N(\mathfrak{C}) \leq B} \mu(\mathfrak{C}) N(\mathfrak{C})^{d-1-n} \leq \zeta_{K}(n+1-d)=O(1) .
$$

Since $d \geq 2$, (19) follows. Similarly,

$$
\begin{aligned}
& \sum_{N(\mathfrak{A}) \leq B} N(\mathfrak{A})^{b(n-1, d-1)-d} \sum_{\mathfrak{C} \mid \mathfrak{A}} \mu(\mathfrak{C}) N(\mathfrak{C})^{d-n} \\
& =O\left(B^{1+b(n-1, d-1)-d}\right)=O\left(B^{b(n-1, d-1)-1}\right) .
\end{aligned}
$$

Proposition 2 now follows from Lemma 17.

Proof of Theorem 1. By Lemma 5, §6, and Propositions 1 and 2, Theorem 1 is true for $d \geq n / 2$. But by symmetry, this implies Theorem 1 is true for all $d=1,2, \ldots, n-1$.

\section{REFERENCES}

1. M. Auslander and D. Buchsbaum, Groups, rings, modules, Harper's Series in Modern Mathematics, Harper \& Row, New York, 1974.

2. J. Cassels, An introduction to the geometry of numbers, Grundlehren Math. Wiss., vol. 99, Springer-Verlag, Berlin, 1959. 
3. G. Folland, Real analysis, Wiley, New York, 1984.

4. J. Franke, Y. Manin, and Y. Tschinkel, Rational points of bounded height on Fano varieties, Invent. Math. 95 (1989), 421-435.

5. G. Hardy and E. Wright, An introduction to the theory of numbers, Clarendon Press, Oxford, 1954.

6. E. Hecke, Lectures on the theory of algebraic numbers, Graduate Texts in Math., vol. 77, Springer-Verlag, Berlin, 1981.

7. W. Hodge and D. Pedoe, Methods of algebraic geometry, Cambridge Univ. Press, Cambridge, 1947.

8. S. Lang, Algebraic number theory, Graduate Texts in Math., vol. 110, Springer-Verlag, Berlin, 1986.

9. K. Mahler, Ein Übertragungsprinzip für Konvexe Korper, Časopis Pěst. Mat. Fys. 68 (1939), 93-102.

10. S. Schanuel, Heights in number fields, Bull. Soc. Math. France 107 (1979), 433-449.

11. W. Schmidt, On heights of algebraic subspaces and Diophantine approximation, Ann. of Math. (2) 85 (1967), 430-472.

12. __ Asymptotic formulae for point lattices of bounded determinant and subspaces of bounded height, Duke Math. J. 35 (1968), 327-339.

13. J. Thunder, The number of solutions of bounded height to a system of linear equations (to appear).

Department of Mathematics, University of Michigan, Ann Arbor, Michigan 481091003 